ESAIM: PROCEEDINGS, July 2007, Vol.18, 99-119

Jean-Frédéric Gerbeau \& Stéphane Labbé, Editors

\title{
ASYMPTOTICS OF HIGHER ORDER ENTROPIES
}

\author{
VINCENT GIOVANGIGLI ${ }^{1}$
}

\begin{abstract}
Résumé. Les entropies d'ordre supérieur sont des estimateurs d'entropie cinétique pour les modèles fluides. Ces quantités sont quadratiques en les dérivées de la vitesse $v$ et la température $T$ avec des coefficients dépendants de $T$. On s'intéresse aux développements asymptotiques des entropies d'ordre supérieur lorsque les nombres de Knudsen $\epsilon_{\mathrm{K}}$ et de Mach $\epsilon_{\mathrm{M}}$ sont petits. Les inégalités entropiques correspondantes sont établies lorsque $\|\log T\|_{B M O}+\epsilon_{\mathrm{M}}\|v / \sqrt{T}\|_{L^{\infty}}$ est assez petite, pourvu que la dépendance de la conductivité thermique $\lambda$ et de la viscosité $\eta$ en la température soit celle de la théorie cinétique. Comme exemple d'application, on établit un résultat d'existence de solutions lorsque le nombre de Mach est faible.
\end{abstract}

\begin{abstract}
Higher order entropies are kinetic entropy estimators for fluid models. These quantities are quadratic in the velocity $v$ and temperature $T$ derivatives and have temperature dependent coefficients. We investigate asymptotic expansions of higher order entropies for incompressible flows in terms of the Knudsen $\epsilon_{\mathrm{K}}$ and Mach $\epsilon_{\mathrm{M}}$ numbers. The correspoding entropic inequalities are obtained when $\|\log T\|_{B M O}+\epsilon_{\mathrm{M}}\|v / \sqrt{T}\|_{L^{\infty}}$ is small enough, provided that the temperature dependence of the thermal conductivity $\lambda$ and the viscosity $\eta$ is that given by the kinetic theory. As an example of application of higher order entropic estimates we establish an existence theorem for small Mach number flows.
\end{abstract}

\section{VERSION FranÇAISE RÉSUmÉE}

La notion d'entropie joue un rôle fondamental dans la modélisation des fluides d'un point de vue à la fois physique et mathématique $[2,5,7,9,16,18,22]$. Nous avons introduit dans une étude précédente $[10,11]$ des estimateurs d'entropie cinétique pour les modèles fluides, suggérés par le développement de Enskog. On a établi que ces quantités vérifient des inégalités entropiques conditionnelles dans le cas des fuides incompressibles emplissant l'espace.

Dans cette étude, on s'intéresse aux développements asymptotiques des estimateurs d'entropie cinétique et aux inégalités entropiques correspondantes lorsque les nombres de Mach $\epsilon_{\mathrm{M}}$ et de Knudsen $\epsilon_{\mathrm{K}}$ sont petits. En comparaison, bien que les entropies d'ordre supérieur soient suggérées par le développement de Enskog, on a seulement considéré dans les études précédentes $[10,11]$ des systèmes d'équations aux dérivées partielles dont les coefficients sont d'ordre unité.

Dans la Section 1 nous résumons tout d'abord l'origine des entropies d'ordre supérieur qui sont des estimateurs d'entropie cinétique pour les modèles fluides. Les équations de bilan correspondantes peuvent être interprétées comme une généralisation des équations de Bernstein aux systèmes d'équations aux dérivées partielles utilisant des variables renormalisées [11].

Nous introduisons dans la Section 2 les variables réduites et les petits paramètres naturellement associés aux modèles fluides, notamment les nombres de Mach $\epsilon_{\mathrm{M}}$ et de Knudsen $\epsilon_{\mathrm{K}}$. A l'aide des variables réduites, les équations fluides et les entropies d'ordre supérieur sont adimensionnées et écrites en terme des nombres $\epsilon_{\mathrm{M}}$ et $\epsilon_{\mathrm{K}}$.

${ }^{1}$ CMAP-CNRS, Ecole Polytechnique, 91128 Palaiseau Cedex, France, vincent.giovangigli@polytechnique.edu

(c) EDP Sciences, SMAI 2007 
On introduit également les coordonées moléculaires - associées au temps de collision entre particules et au libre parcours moyen - qui sont telles que tous les petits paramètres sont éliminés des équations correspondantes.

Dans la Section 3 nous résumons des inégalités avec poids dans les espaces de Sobolev et Lebesgue [11]. Ces inégalités sont nécessaires car on utilise des variables renormalisées par des puissances de la température ainsi que des coefficients de transport qui dépendent de la température. On précise également comment ces inégalités sont transformées par un changement d'échelle des coordonnées.

Dans la Section 4 on s'intéresse aux entropies d'ordre supérieur lorsque les paramètres naturels des modèles fluides qui interviennent dans les équations de conservation $\epsilon_{\mathrm{M}}$ et $\epsilon_{\mathrm{K}}$ sont petits. Les estimations correspondantes sont obtenues lorsque la quantité $\chi=\|\log T\|_{B M O}+\epsilon_{\mathrm{M}}\|v / \sqrt{T}\|_{L^{\infty}}$ est assez petite, pourvu que la dépendance de la conductivité thermique $\lambda$ et de la viscosité $\eta$ en la température $T$ soit celle de la théorie cinétique, c'est-à-dire essentiellement de la forme $T^{\varkappa}$ avec $\varkappa \geq 1 / 2$. Il est important de noter que cette quantité $\chi$ est petite pour les faibles nombres de Mach car on a $\chi=\mathcal{O}\left(\epsilon_{\mathrm{M}}\right)$ et elle est également invariante par changement d'échelle. Dans la situation du scaling logarithmique, on montre aussi que les entropies d'ordre supérieur sont proches des normes de Sobolev de l'entropie fluide en variables moléculaires.

Dans la Section 5, nous présentons un exemple d'application des inégalités entropiques d'ordre supérieur. Nous établissons un théorème d'existence dans la limite des faibles nombres de Mach $\epsilon_{\mathrm{M}}$ et de Knudsen $\epsilon_{\mathrm{K}}$. Par commodité, on condidère tout d'abord les équations écrites en coordonnées moléculaires et on renforce un théorème obtenu dans [11]. On obtient en particulier des estimations a priori des correcteurs d'entropie cinétique sous la forme de puissances du nombre de Knudsen. Par transformation en coordonnées macroscopiques on obtient alors un théorème d'existence globale pour les faibles nombres de Mach et Knudsen.

\section{INTRODUCTION}

The notion of entropy has been shown to be of fundamental importance in fluid modeling from both a physical and mathematical point of view $[1,2,5-7,9,14,16,18,22]$. We have introduced in a previous work [10,11] a notion of kinetic entropy estimators for fluid models, suggested by Enskog expansion of kinetic entropy. Conditional entropic inequalities have been established for these quantities in the situation of incompressible flows spanning the whole space.

In this paper, we further investigate asymptotic expansions of these higher order entropies and we study the corresponding conditional entropic inequalities when the Mach number $\epsilon_{\mathrm{M}}$ and the Knudsen number $\epsilon_{\mathrm{K}}$ are small. In contrast, although higher order entropies are suggested by Enskog expansion, only fluid systems with coefficients of order unity were considered in previous work.

In Section 1 we first summarize the mathematical and physical motivations for higher order entropies which are kinetic entropy estimators for fluid models. The corresponding balance equations may be seen as a generalization of Bernstein equation to systems of partial differential equations associated with renormalized variables [11].

We introduce in Section 2 the natural rescaled variables and small parameters associated with fluid models, in particular the Mach number $\epsilon_{\mathrm{M}}$ and the Knudsen number $\epsilon_{\mathrm{K}}$. Thanks to the rescaled variables, the governing equations and the higher order entropies are rewriten in terms of $\epsilon_{\mathrm{M}}$ and $\epsilon_{\mathrm{K}}$. We also introduce the molecular coordinates - associated with the particle collision time and the mean free path - which are such that all small parameters are eliminated from the corresponding system of partial differential equations.

In Section 3 we summarize some weighted inequalities in Sobolev and Lebesgue spaces [11]. These inequalities are required since we are using renormalized variables with powers of temperature as weights and since we also consider flows with temperature dependent thermal conductivity and viscosity. We further discuss how the various inequalities are transformed by a change of scale in the coordinate system.

We investigate in Section 4 higher order entropies when the natural small parameters of fluid models are taken into account in the governing equations. The corresponding entropic estimates are obtained when the quantity $\chi=\|\log T\|_{B M O}+\epsilon_{\mathrm{M}}\|v / \sqrt{T}\|_{L^{\infty}}$ is small enough, provided that the temperature dependence of thermal conductivity and viscosity is that given by the kinetic theory, that is, essentially in the form of a power law of temperature $T^{\varkappa}$ with $\varkappa \geq 1 / 2$. Note that the quantity $\chi$ is small when the Mach number is small since we formally have $\chi=\mathcal{O}\left(\epsilon_{\mathrm{M}}\right)$ and $\chi$ is also scaling invariant. In the situation of logarithmic scaling the kinetic 
entropy estimators are also shown to be closely associated with Sobolev norms of the fluid entropy in molecular coordinates.

In Section 5, as an example of application, we investigate an existence theorem in the limit of small Knudsen $\epsilon_{\mathrm{K}}$ and Mach $\epsilon_{\mathrm{M}}$ numbers. For convenience we first consider the fluid equations written in molecular coordinates and strenghen an existence result previously obtained in [11]. We obtain in particular a priori bounds on kinetic entropy correctors in the form of powers of the Knudsen number. Upon restoring the macroscopic time and space scale we obtain a global existence theorem for small Mach and Knudsen number flows.

\section{Higher order ENTROPIES}

In this section we briefly motivate the introduction of higher order entropies by discussing Bernstein method and inspecting Enskog expansion of kinetic entropy [10,11]

\subsection{A thermodynamic interpretation of Bernstein equation}

For parabolic - or elliptic - scalar equations, a priori gradient estimates can be obtained by using Bernstein method [17]. More specifically, consider - as a simple exemple - the heat equation

$$
\partial_{t} u-\Delta u=0
$$

Defining $\zeta=\left|\partial_{x} u\right|^{2}=\partial_{x} u \cdot \partial_{x} u$, we then have Bernstein equation

$$
\partial_{t} \zeta-\Delta \zeta+2\left|\partial_{x}^{2} u\right|^{2}=0
$$

where $\left|\partial_{x}^{2} u\right|^{2}=\partial_{x}^{2} u: \partial_{x}^{2} u=\sum_{i j}\left(\partial_{i j} u\right)^{2}$. The structure of (1) then appears to be formally similar to that of an entropy balance, where $\zeta$ plays the rôle of a generalized entropy, even though there also exist zeroth order entropies like $u^{2}$. In the next section, we introduce a kinetic framework supporting this entropic interpretation.

\subsection{Enskog second order expansion of kinetic entropy}

The state of a monatomic gas is described by a particle distribution function $f(t, x, c)$-governed by Boltzmann equation - where $t$ denotes time, $x$ the $n$-dimensional spatial coordinate, and $c$ the particle velocity $[2,5,7,9]$. Approximate solutions of Boltzmann's equation can be obtained from a first order Enskog expansion $f=f^{(0)}\left(1+\varepsilon \phi^{(1)}+\mathcal{O}\left(\varepsilon^{2}\right)\right)$ where $f^{(0)}$ is the local Maxwellian distribution, $\phi^{(1)}$ the perturbation associated with the Navier-Stokes regime and $\varepsilon$ the usual Enskog formal expansion parameter. The compressible Navier-Stokes equations can then be obtained upon taking moments of Boltzmann's equation [3, 7,9].

The kinetic entropy $S^{\text {kin }}=-k_{\mathrm{B}} \int_{\mathbb{R}^{n}} f(\log f-1) d c$, where $k_{\mathrm{B}}$ denotes Boltzmann constant, satisfies the $H$ theorem, that is, the second principle of thermodynamics. The expansion of $S^{\text {kin }}$ induced by a second order Enskog expansion can be written

$$
S^{\mathrm{kin}}=S^{(0)}+\varepsilon^{2} S^{(2)}+\mathcal{O}\left(\varepsilon^{3}\right),
$$

where $S^{(0)}$ is the usual zeroth order macroscopic entropy evaluated from the Maxwellian distribution $f^{(0)}$ and $S^{(2)}=-k_{\mathrm{B}} / 2 \int_{\mathbb{R}^{n}}\left(\phi^{(1)}\right)^{2} f^{(0)} d c$. For compressible monatomic gases one can establish that [11]

$$
-\rho S^{(2)}=\bar{\lambda}\left|\partial_{x} T\right|^{2}+\frac{1}{2} \bar{\eta}|d|^{2}
$$

where $T$ denotes the absolute temperature, $\rho$ the density, $v$ the gas velocity, $d=\partial_{x} v+\partial_{x} v^{t}-\frac{2}{n}\left(\partial_{x} \cdot v\right) I$ the strain rate tensor and $|d|^{2}=\sum_{i j} d_{i j}^{2}$, and where the scalar coefficients $\bar{\lambda}$ and $\bar{\eta}$ only depend on temperature. In a first approximation, using a single term in orthogonal polynomial expansions of perturbed distribution functions, one can establish that $\bar{\lambda}=\left(1 / 2 r c_{p}\right) \lambda^{2} / T^{3}$ and $\bar{\eta}=(1 / 2 r) \eta^{2} / T^{2}$ where $c_{p}$ is the constant pressure specific heat per unit mass, $r$ the gas constant per unit mass, $\lambda$ the thermal conductivity, $\eta$ the shear viscosity, and the actual values of the numerical factors in front of $\bar{\lambda}$ and $\bar{\eta}$ are evaluated here for $n=3$. 


\subsection{Enskog higher order expansion of kinetic entropy}

More generally, higher order Enskog expansions $f / f^{(0)}=1+\varepsilon \phi^{(1)}+\cdots+\epsilon^{2 k} \phi^{(2 k)}+\mathcal{O}\left(\varepsilon^{2 k+1}\right)$ induce higher order expansions for $S^{\mathrm{kin}}$

$$
S^{\mathrm{kin}}-S^{(0)}=\varepsilon^{2} S^{(2)}+\varepsilon^{3} S^{(3)}+\cdots+\varepsilon^{2 k} S^{(2 k)}+\mathcal{O}\left(\varepsilon^{2 k+1}\right),
$$

where $S^{(l)}$ is a sum of terms in the form $k_{\mathrm{B}} \int_{\mathbb{R}^{n}} \prod_{1 \leq i \leq l}\left(\phi^{(i)}\right)^{\nu_{i}} f^{(0)} d c$ with nonnegative integers $\nu_{i} \geq 0,1 \leq i \leq l$, such that $l=\sum_{1 \leq i \leq l} i \nu_{i}$. From the general expression of $\phi^{(l)}$ in the absence of external forces acting on the particles, one can establish that

$$
S^{(2 k)}=\rho r\left(\frac{\eta}{\rho \sqrt{r T}}\right)^{2 k} \sum_{\nu} c_{\nu} \prod_{1 \leq|\alpha| \leq 2 k}\left(\frac{\partial_{x}^{\alpha} T}{T}\right)^{\nu_{\alpha}}\left(\frac{\partial_{x}^{\alpha} \rho}{\rho}\right)^{\nu_{\alpha}^{\prime}}\left(\frac{\partial_{x}^{\alpha} v}{\sqrt{r T}}\right)^{\nu_{\alpha}^{\prime \prime}}
$$

where $\nu_{\alpha}, \nu_{\alpha}^{\prime}, \nu_{\alpha}^{\prime \prime} \in \mathbb{N}, \alpha \in \mathbb{N}^{n}$, and $\nu=\left(\nu_{\alpha}, \nu_{\alpha}^{\prime}, \nu_{\alpha}^{\prime \prime}\right)_{1 \leq|\alpha| \leq 2 k}$ must be such that $\sum_{1 \leq|\alpha| \leq 2 k}|\alpha|\left(\nu_{\alpha}+\nu_{\alpha}^{\prime}+\nu_{\alpha}^{\prime \prime}\right)=2 k$ and where the coefficients $c_{\nu}$ are smooth scalar functions of $\log T$ of order unity.

After integrations by parts in the integral $\int_{\mathbb{R}^{n}} S^{(2 k)} d x$, in order to eliminate spatial derivatives of order strictly greater than $k$, and by using interpolation inequalities, one obtains that $\left|\int_{\mathbb{R}^{n}} S^{(2 k)} d x\right|$ is essentially controled by the integral of

$$
\gamma^{[k]}=r \rho\left(\frac{\eta}{\rho \sqrt{r T}}\right)^{2 k}\left(\left|\frac{\partial_{x}^{k} T}{T}\right|^{2}+\left|\frac{\partial_{x}^{k} \rho}{\rho}\right|^{2}+\left|\frac{\partial_{x}^{k} v}{\sqrt{r T}}\right|^{2}\right),
$$

or equivalently of

$$
\widetilde{\gamma}^{[k]}=r \rho\left(\frac{\eta}{\rho \sqrt{r T}}\right)^{2 k}\left(\left|\partial_{x}^{k} \log T\right|^{2}+\left|\partial_{x}^{k} \log \rho\right|^{2}+\left|\partial_{x}^{k}(v / \sqrt{r T})\right|^{2}\right),
$$

and $\left|\int_{\mathbb{R}^{n}} S^{(2 k-1)} d x\right|$ is also controled by $\int_{\mathbb{R}^{n}} \gamma^{[k]} d x$ and $\int_{\mathbb{R}^{n}} \gamma^{[k-1]} d x$. This suggests the quantities $\gamma^{[k]}$ or $\widetilde{\gamma}^{[k]}$ as $(2 k)^{\text {th }}$ order kinetic entropy correctors - or kinetic entropy deviation estimators [11]. Note that, at variance with $S^{(2)}$, it is not clear that $S^{(2 k)}$ has a sign, and this is a motivation for using $\gamma^{[k]}$ and $\widetilde{\gamma}^{[k]}$ rather than $S^{(2 k)}$, beyond simplicity. We are therefore looking for majorizing entropic correctors, and the fact that entropic inequalities can be obtained for such correctors is a consequence of the structure of the fluid equations. A similar analysis can also be conducted for the Fisher information and suggests the same quantities $\gamma^{[k]}$ or $\widetilde{\gamma}^{[k]}$ as higher order kinetic information correctors.

Remarque 1.1. Since the kinetic entropy correctors $\gamma^{[k]}$ or $\widetilde{\gamma}^{[k]}$ are only majorizing correctors, we are free to modify these quantities for convenience. These estimators generally have to be adapted to the mathematical structure of the fluid equations under consideration. In particular, for hyperbolic-parabolic systems of partial differential equations, perturbations are needed for hyperbolic variables [12]. These correctors may also be rescaled by mutiplicative constants depending on $k[11,12]$. The temperature dependence of higher order entropy estimators may also be simplified when the temperature dependence of transport coefficient is simplified.

\subsection{Persistence of kinetic entropy versus extended fluid models}

Denoting by $\gamma^{[0]}$ a nonnegative quantity associated with the zeroth order entropy $S^{(0)}$, we will investigate entropicity properties of kinetic entropy estimators in the form $\Gamma^{[l]}=\gamma^{[0]}+\cdots+\gamma^{[l]}$, with $0 \leq l \leq k$, for the solutions of a second order system of partial differential equations modeling a fluid. For this fluid system, the zeroth order entropy $S^{(0)}$ is already of fundamental importance as imposed by the hyperbolic-parabolic structure of these equations $[9,13,16]$. We only consider the quantities $\gamma^{[0]}+\cdots+\gamma^{[l]}, 0 \leq l \leq k$, as a family of mathematical entropy estimators - of kinetic origin - and we will establish that they indeed satisfy conditional entropic principles for solutions of Navier-Stokes type equations. Higher order entropic estimates may also be seen as a generalization of Bernstein method for systems of partial differential equations but involving renormalized variables. 
This point of view differs from that of thermodynamic theories that have already considered entropies differing from that of zeroth order, that is, entropies depending on macroscopic variable gradients. These generalized entropies have been associated notably with Burnett type equations or extended thermodynamics. In both situations, new macroscopic equations are correspondingly obtained, that is, 'extended fluid models', which are systems of partial differential equations of higher orders than Navier-Stokes type equations.

\section{NONDIMENSIONALIZATION}

We introduce in this section the rescaled fluid variables, the rescaled fluid equations, and the natural small parameters needed to investigate asymptotic expansions of higher order entropies.

\subsection{Rescaled variables}

In order to investigate asymptotic expansions of higher order entropies, we need to specify the order of magnitude of the various terms appearing in fluid governing equations. To this purpose, for each quantity $\phi$, we introduce a typical order of magnitude denoted by $\langle\phi\rangle$. In particular, we introduce a characteristic length $\langle x\rangle$, velocity $\langle v\rangle$, density $\langle\rho\rangle$, viscosity $\langle\eta\rangle$, and pressure $\langle p\rangle$. The order of magnitude of the sound velocity $c$ is then $\langle c\rangle^{2}=\langle p\rangle \mid\langle\rho\rangle$ and from the state law we also have $\langle c\rangle^{2}=\langle r\rangle\langle T\rangle$ where $r$ denotes the gas constant per unit mass and $T$ the absolute temperature, and the Reynolds number is given by

$$
\operatorname{Re}=\frac{\langle\rho><v><x>}{<\eta>} .
$$

An important aerodynamic length upon consideration is the dissipation length $\langle x\rangle^{\text {dis }}$ defined such that the corresponding Reynolds number is unity $\langle x\rangle^{\text {dis }}=\langle\eta\rangle /\langle\rho\rangle\langle v\rangle$ and we can then write $\operatorname{Re}=\langle x\rangle /\langle x\rangle^{\text {dis }}$. We define the characteristic time from the characteristic length $\langle x\rangle$ and the characteristic velocity $\langle v\rangle$ by letting $\langle t\rangle=\langle x\rangle /\langle v\rangle$.

We also introduce a typical mean free path $\langle l\rangle$ and from kinetic theory $\langle\eta\rangle=\langle\rho\rangle\langle c\rangle\langle l\rangle$ [7]. Denoting by $\epsilon_{\mathrm{K}}$ the Knudsen number $\langle l\rangle /\langle x\rangle$ and $\epsilon_{\mathrm{M}}$ the Mach number $\langle v\rangle /\langle c\rangle$ we then have the Von Karman relation

$$
\epsilon_{\mathrm{M}}=\frac{\langle v>}{<c>}=\frac{<l>}{<x>} \operatorname{Re}=\epsilon_{\mathrm{K}} \operatorname{Re},
$$

which relates $\epsilon_{\mathrm{K}}$ and $\epsilon_{\mathrm{M}}$. We will assume in the following that the Knudsen number $\epsilon_{\mathrm{K}}$ is small and the Mach number $\epsilon_{\mathrm{M}}$ will also be small, since we are especially interested in flows where the characteristic length $\langle x\rangle$ is the dissipative length $\langle x\rangle^{\text {dis }}$ and the Reynolds number is then unity.

Upon defining the reduced quantity $\widehat{\phi}=\phi /\langle\phi\rangle$ associated with each quantity $\phi$ of the fluid model, we can now estimate the order of magnitude of each term in the governing partial differential equations and in the definition of higher order entropies. We will assume, for the sake of simplicity, that we have $\hat{r}=1$ so that $\widehat{c}^{2}=\widehat{T}$ in particular.

\subsection{Rescaled governing equations}

We will only consider incompressible flows spanning the whole space that are 'constant at infinity'. The case of compressible flows [12] or zero Mach number flows as well as other boundary conditions are beyond the scope of the present paper. The equations governing incompressible flows can be written in the form [18]

$$
\begin{gathered}
\partial_{x} \cdot v=0 \\
\partial_{t}(\rho v)+\partial_{x} \cdot(\rho v \otimes v)+\partial_{x} p+\partial_{x} \cdot \Pi=0 \\
\partial_{t}(\rho e)+\partial_{x} \cdot(\rho e v)+\partial_{x} \cdot \mathcal{Q}=-\Pi: \partial_{x} v
\end{gathered}
$$

where $t$ is time, $x$ the $n$-dimensional spatial coordinate, $\rho$ the mass density, $v$ the velocity vector, $p$ the pressure, $\Pi$ the viscous tensor, $e$ the internal energy per unit mass, and $\mathcal{Q}$ the heat flux. The viscous tensor is given by 
$\Pi=-\eta d$ where $\eta$ is the shear viscosity and $d$ the strain rate tensor $d=\partial_{x} v+\partial_{x} v^{t}$, and the heat flux is given by $\mathcal{Q}=-\lambda \partial_{x} T$ where $\lambda$ is the thermal conductivity. For the sake of simplicity the internal energy per unit mass $e$ is taken in the form $e=c_{v} T$ where $c_{v}$ is a constant.

Upon using the general notation of Section 2.1 and letting $\widehat{\rho}=1$ thanks to incompressibility the reduced equations can then be written

$$
\begin{aligned}
& \partial_{\widehat{x}} \cdot \widehat{v}=0, \\
& \partial_{\widehat{t}} \widehat{v}+\partial_{\widehat{x}} \cdot(\widehat{v} \otimes \widehat{v})+\frac{1}{\epsilon_{M}^{2}} \partial_{\widehat{x}} \widehat{p}+\frac{1}{\operatorname{Re}} \partial_{\widehat{x}} \cdot \widehat{\Pi}=0, \\
& \partial_{\widehat{t}} \widehat{e}+\partial_{\widehat{x}} \cdot(\widehat{v} \widehat{e})+\frac{1}{\operatorname{Re}} \partial_{\widehat{x}} \cdot \widehat{\mathcal{Q}}=-\frac{\epsilon_{\mathrm{N}}^{2}}{\operatorname{Re}} \widehat{\Pi}: \partial_{\widehat{x}} \widehat{v} .
\end{aligned}
$$

Note that, in contrast with the rescaled system (13)-(15), all systems of partial differential equations previously considered in $[10,11]$ did not contain any small parameter. By defining $\widehat{p}_{2}=\left(\widehat{p}-\widehat{p}_{0}\right) / \epsilon_{\mathrm{M}}^{2}$ we may also replace $\frac{1}{\epsilon_{\mathrm{M}}^{2}} \partial_{\widehat{x}} \widehat{p}$ by $\partial_{\widehat{x}} \widehat{p}_{2}$ in the momentum governing equation (14).

\subsection{Rescaled higher order entropies}

Specializing formally the expression (6) for $\gamma^{[k]}$ to the situation of incompressible flows the derivatives with respect to the mass density $\rho$ vanish. Further letting $\left\langle\gamma^{[k]}\right\rangle=\langle\rho r\rangle=\langle\rho\rangle\langle r\rangle$ we deduce after some algebra, using $\hat{r}=1$ and $\hat{\rho}=1$, that the rescaled higher order entropy correctors $\widehat{\gamma}^{[k]}=\gamma^{[k]} /\langle\rho\rangle\langle r\rangle$ are given by

$$
\widehat{\gamma}^{[k]}=\epsilon_{\mathrm{K}}^{2 k}\left(\frac{\widehat{\eta}}{\sqrt{\widehat{T}}}\right)^{2 k}\left\{\left|\frac{\partial_{\widehat{x}}^{k} \widehat{T}}{\widehat{T}}\right|^{2}+\epsilon_{\mathrm{M}}^{2}\left|\frac{\partial_{\widehat{x}}^{k} \widehat{v}}{\sqrt{\widehat{T}}}\right|^{2}\right\},
$$

and we have recovered that the $(2 k)^{\text {th }}$ order entropy correctors are formally of order $\mathcal{O}\left(\epsilon_{\mathrm{K}}^{2 k}\right)$ as was expected from their construction in Section 1.

At zeroth order, we will use for convenience the modified entropy $\rho\left(e-e_{\infty}+\frac{1}{2} v \cdot v\right) / T_{\infty}-\left(S^{(0)}-S_{\infty}^{(0)}\right)$ so that we obtain after rescaling

$$
\widehat{\gamma}^{[0]}=\left(\frac{\widehat{e}-\widehat{e}_{\infty}}{\widehat{T}_{\infty}}-\left(\widehat{s}-\widehat{s}_{\infty}\right)\right)+\epsilon_{\mathrm{M}}^{2} \frac{\widehat{v}^{2}}{2 \widehat{T}_{\infty}} .
$$

The rescaled kinetic entropy estimators $\widehat{\Gamma}^{[k]}$ are finally given by $\widehat{\Gamma}^{[k]}=\widehat{\gamma}^{[0]}+\cdots+\widehat{\gamma}^{[k]}$ and similar definitions can be introduced with respect to the higher order entropies $\widetilde{\gamma}^{[k]}(7)$.

\subsection{Molecular coordinates}

The proper framework required to investigate asymptotic expansions of higher order entropies involve the rescaled coordinates $(\widehat{t}, \widehat{x})$, the rescaled unknowns $(\widehat{v}, \widehat{T})$, and the rescaled governing equations (13)-(15) depending on the small parameters $\epsilon_{\mathrm{K}}$ and $\epsilon_{\mathrm{M}}$. In this framework, parameter dependent a priori estimates and entropic inequalities can directly be obtained from the rescaled fluid governing equations.

On the other hand, it is also possible to completely eliminate the parameters $\epsilon_{\mathrm{K}}$ and $\epsilon_{\mathrm{M}}$ from the governing equations and from higher order entropy expansions by using a set of coordinates associated with the molecular properties of the fluid. More specifically, let us introduce the new coordinates

$$
\bar{x}=\frac{\widehat{x}}{\epsilon_{\mathrm{K}}}, \quad \bar{t}=\frac{\widehat{t}}{\epsilon_{\mathrm{K}} \epsilon_{\mathrm{M}}},
$$

associated with the characteristic length $\epsilon_{\mathrm{K}}\langle x\rangle=\langle l\rangle$ and the characteristic time $\epsilon_{\mathrm{K}} \epsilon_{\mathrm{M}}\langle t\rangle=\langle l\rangle /\langle c\rangle$ so that $\bar{x}$ is measured in units of the mean free path $\langle l\rangle$ and $\bar{t}$ in units of the particle collision time $\langle l\rangle /\langle c\rangle$. We then have $\partial_{\bar{x}}^{\alpha}=\epsilon_{\mathrm{K}}^{|\alpha|} \partial_{x}^{\alpha}$ and $\partial_{\bar{t}}^{\sigma}=\left(\epsilon_{\mathrm{K}} \epsilon_{\mathrm{M}}\right)^{\sigma} \partial_{t}^{\sigma}$ for any multiindex $\alpha \in \mathbb{N}^{n}$ and any $\sigma \in \mathbb{N}$. By using these 
molecular coordinates $(\bar{t}, \bar{x})$, and upon defining the velocity $\bar{v}$ by

$$
\bar{v}=\epsilon_{\mathrm{M}} \widehat{v}=\frac{v}{<c\rangle}
$$

we obtain after a little algebra the governing equations

$$
\begin{gathered}
\partial_{\bar{x}} \cdot \bar{v}=0, \\
\partial_{\bar{t}} \bar{v}+\partial_{\bar{x}} \cdot(\bar{v} \otimes \bar{v})+\partial_{\bar{x}} p+\frac{1}{\operatorname{Re}} \partial_{\bar{x}} \cdot \bar{\Pi}=0, \\
\partial_{\bar{t}} e+\partial_{\bar{x}} \cdot(\bar{v} e)+\frac{1}{\operatorname{Re}} \partial_{\bar{x}} \cdot \overline{\mathcal{Q}}=-\frac{1}{\operatorname{Re}} \bar{\Pi}: \partial_{\bar{x}} \bar{v}
\end{gathered}
$$

where $\bar{\Pi}=-\eta \bar{d}, \bar{d}=\partial_{\bar{x}} \bar{v}+\partial_{\bar{x}} \bar{v}^{t}$ and $\overline{\mathcal{Q}}=-\lambda \partial_{\bar{x}} T$. In these equations, with a slight abuse of motation, we have denoted by the same letter the corresponding functions in physical $x$ or molecular $\bar{x}$ coordinates. It is then remarkable that all small parameters have been eliminated from the system (20)-(22) so that we may use any result obtained in previous work $[10,11]$. A second method to investigate asymptotic expansions is therefore to use molecular coordinates and to map back these results to the unknowns $(v, T)$ written in terms of the macroscopic coordinates $(\widehat{t}, \widehat{x})$. These two methods are of course equivalent and both methods will be used in the following.

\subsection{Temperature dependence of transport coefficients}

Previous analyses have shown that it is necessary to take into account the temperature dependence of transport coefficients $[10,11]$. The kinetic theory of gases and the minimum principle for temperature lead to the following simplified assumptions concerning the temperature dependence of the thermal conductivity $\lambda(T)$ and the shear viscosity $\eta(T)$. We assume that $\lambda$ and $\eta$ are $C^{\infty}(0, \infty)$ and such that there exist $\varkappa, \underline{\mathfrak{a}}>0, \overline{\mathfrak{a}}>0$, and $\overline{\mathfrak{a}}_{\sigma}>0$ for any $\sigma \geq 1$, with

$$
\underline{\mathfrak{a}} T^{\varkappa} \leq \lambda / c_{v} \leq \overline{\mathfrak{a}} T^{\varkappa}, \quad \underline{\mathfrak{a}} T^{\varkappa} \leq \eta \leq \overline{\mathfrak{a}} T^{\varkappa}, \quad T^{\sigma}\left(\left|\partial_{T}^{\sigma} \lambda\right|+\left|\partial_{T}^{\sigma} \eta\right|\right) \leq \overline{\mathfrak{a}}_{\sigma} T^{\varkappa}
$$

Kinetic theory suggests that $1 / 2 \leq \varkappa \leq 1$ but the situations where $0 \leq \varkappa<1 / 2$ or $\varkappa>1$ are still interesting to investigate from a mathematical point of view. Under these simplifying assumptions, we may accordingly simplify the temperature dependence of higher order kinetic correctors which can be taken in the form

$$
\widehat{\gamma}^{[k]}=\epsilon_{\mathrm{K}}^{2 k} \frac{1}{\widehat{T}^{k(1-2 \varkappa)}}\left\{\left|\frac{\partial_{\widehat{x}}^{k} \widehat{T}}{\widehat{T}}\right|^{2}+\epsilon_{\mathrm{M}}^{2}\left|\frac{\partial_{\widehat{x}}^{k} \widehat{v}}{\sqrt{\widehat{T}}}\right|^{2}\right\},
$$

with similar simplifications for entropy correctors in the form (7).

\section{Weighted INEQUALities}

Higher order entropies naturally introduce weight factors in the form of powers of temperature when estimating Lebesgue norms of the flow variables derivatives. Temperature weights also arise when investigating fluid models with temperature dependent transport coefficients. We restate here weighted estimates of derivatives [11] and further investigate the scaling dependence of the corresponding estimating constants.

\subsection{The Muckenhoupt condition}

A natural condition associated with weights has been shown to be the Muckenhoupt property $A_{p}$, where $1 \leq p \leq \infty[8,19,20]$ 
Définition 3.1. Let $g \in L_{l o c}^{1}\left(\mathbb{R}^{n}\right)$ be positive and locally integrable and let $1<p<\infty$. We say that $g$ satisfies the Muckenhoupt condition $A_{p}$ if

$$
[g]_{A_{p}}=\sup _{Q}\left(\frac{1}{|Q|} \int_{Q} g d x\right)\left(\frac{1}{|Q|} \int_{Q} g^{-\frac{1}{p-1}} d x\right)^{p-1}<\infty
$$

where the supremum is taken over all cubes.

For detailed studies about the Muckenhoupt property we refer to the book of Garcia-Cuerva and Rubio de Francia [8]. We have in particular $A_{p} \cap A_{q}=A_{\min (p, q)}$ and the weights of $A_{p}$ have their logarithms in BMO $[8,19]$. A locally summable function $f$ belongs to the space $B M O\left(\mathbb{R}^{n}\right)$ if

$$
\|f\|_{B M O}=\sup _{Q} \frac{1}{|Q|} \int_{Q}\left|f(x)-\bar{f}_{Q}\right| d x<\infty
$$

where the supremum is taken over all cubes $Q$ and where $\bar{f}_{Q}=1 /|Q| \int_{Q} f(x) d x$ denotes the average of $f$ over $Q$ [19]. The function space $B M O$ has been introduced by John and Nirenberg [15] and naturally arises when estimating the norms of the weighted operators $T^{\theta} R_{i} T^{-\theta}$ where $R_{i}=(-\Delta)^{-1 / 2} \partial_{i}, 1 \leq i \leq n$, are Riesz transforms, or when using the Coifman and Meyer inequalities [20].

Théorème 3.2. There exists constants $b(n)$ and $B(n)$ such that for any $\theta \in \mathbb{R}$, any $u \in B M O$, and any $1<p<\infty$, the condition

$$
|\theta|\|u\|_{B M O}<\frac{1}{2} b(n) \min (1, p-1)
$$

implies that $\exp (\theta u) \in A_{p}$ and

$$
[\exp (\theta u)]_{A_{p}} \leq(1+B(n))^{p} .
$$

Moreover, the constants $b(n)$ and $B(n)$ only depend on $n$ and are thus invariant by a change of scale in the coordinate system.

Proof. These estimates are proved in [11] and the scale invariance of $b(n)$ and $B(n)$ is straightforward since both the $B M O$ seminorm and the $A_{p}$ condition number $[g]_{A_{p}}$ are scale invariant. That is, if $u \in B M O$ and $\tilde{u}(x)=u(\xi x)$ where $\xi>0$ is a rescaling factor, then $\|\tilde{u}\|_{B M O}=\|u\|_{B M O}$ and similarly $[g]_{A_{p}}=[\tilde{g}]_{A_{p}}$ for any $\xi>0$.

We now investigate the continuity of Calderón-Zygmund operators in weighted Lebesgue spaces. In the following theorem the quantitites $c_{0}, c_{1}, c_{2}$ are the constants naturally associated with the norm of a CalderónZygmund operator $\mathcal{G}[19]$.

Théorème 3.3. Let $\mathcal{G}$ be a Calderón-Zygmund operator, let $1<p<\infty$, and let $g$ be a weight in $A_{p}$. Then the operator $\mathcal{G}$ is bounded in $L^{p}(g d x)$, or equivalently, the operator $g^{1 / p} \mathcal{G} g^{-1 / p}$ is bounded in $L^{p}$, with norm lower than $\mathcal{C}\left(c_{0}, c_{1}, c_{2}, n, p,[g]_{A_{p}}\right)$, where $c_{0}, c_{1}, c_{2}$ are the constants naturally associated with the norm of $\mathcal{G}$.

Proof. We refer to the books of Garcia-Cuerva and Rubio de Francia [8] and of Yves Meyer [19].

\subsection{A multilinear estimate}

We investigate weighted multilinear estimates, with weights in $A_{p}$, and we denote by $A\left(\mathbb{R}^{n}\right)$ the Wiener algebra in $\mathbb{R}^{n}$.

Théorème 3.4. Let $k, l$ be positive integers, and $\alpha^{j}, 1 \leq j \leq l$, be multiindices such that $\left|\alpha^{j}\right| \geq 1,1 \leq j \leq l$, and $k=\sum_{1 \leq j \leq l}\left|\alpha^{j}\right|$. Let $1<p<\infty, g \in A_{p}$, and $u_{1}, \ldots, u_{l}$, be such that there exist constants $u_{j, \infty}$ with 
$u_{j}-u_{j, \infty} \in H^{k}\left(\mathbb{R}^{n}\right) \cap A\left(\mathbb{R}^{n}\right)$, and such that $g^{\frac{1}{p}} \partial_{x}^{k} u_{j} \in L^{p}, 1 \leq j \leq l$. There exists a constant $c=c\left(k, n, p,[g]_{A_{p}}\right)$ only depending on $k, n, p$, and $[g]_{A_{p}}$, such that

$$
\left\|g^{1 / p} \prod_{1 \leq j \leq l} \partial_{x}^{\alpha^{j}} u_{j}\right\|_{L^{p}} \leq c\left(\sum_{1 \leq j \leq l}\left\|u_{j}\right\|_{B M O}\right)^{l-1}\left(\sum_{1 \leq j \leq l}\left\|g^{1 / p} \partial_{x}^{k} u_{j}\right\|_{L^{p}}\right)
$$

where we define

$$
\left\|g^{1 / p} \partial_{x}^{k} \mathrm{v}\right\|_{L^{p}}^{p}=\sum_{|\alpha|=k} \frac{k !}{\alpha !} \int_{\mathbb{R}^{n}} g\left|\partial_{x}^{\alpha} \mathrm{v}\right|^{p} d x
$$

using the multinomial coefficients $k ! / \alpha !=k ! / \alpha_{1} ! \cdots \alpha_{n} !$ [4]. Moreover, the constant $c$ is invariant by a change of scale in the coordinate system.

Proof. The existence of the constant $c=c\left(k, n, p,[g]_{A_{p}}\right)$ is established in [11] by using the Coifman-Meyer multilinear theory [20] with Theorems 3.2 and 3.3. The scale invariance of the constant $c$ is easily established by a direct change of variable in (25) since the $B M O$ norms are invariant and since there is an equal number of derivatives $k$ in each member.

Remarque 3.5. The definition of $\left\|\partial_{x}^{k} \mathrm{v}\right\|_{L^{p}}^{p}$ using the multinomial coefficients yields in particular for $p=2$

$$
\left\|\partial_{x}^{k} \mathrm{v}\right\|_{L^{2}}^{2}=\sum_{|\alpha|=k} \frac{k !}{\alpha !} \int_{\mathbb{R}^{n}}\left(\partial_{x}^{\alpha} \mathrm{v}\right)^{2} d x=\sum_{1 \leq i_{1}, \ldots, i_{k} \leq n}\left\|\partial_{i_{1}} \cdots \partial_{i_{k}} \mathrm{v}\right\|_{L^{2}}^{2}
$$

so that it is compatible with the classical definition $\left|\partial_{x}^{2} \mathrm{v}\right|^{2}=\sum_{i j}\left(\partial_{i} \partial_{j} \mathrm{v}\right)^{2}$ already used in Section 1.1. This natural definition also simplifies the analytic form of higher order entropies governing equations.

Remarque 3.6. The space of smooth functions with compact support $\mathcal{D}\left(\mathbb{R}^{n}\right)$ is dense in $H^{k}\left(\mathbb{R}^{n}\right) \cap B M O\left(\mathbb{R}^{n}\right)$ if and only if $k \geq n / 2$. For $k<n / 2, \mathcal{D}\left(\mathbb{R}^{n}\right)$ is neither dense in $H^{k}\left(\mathbb{R}^{n}\right) \cap L^{\infty}\left(\mathbb{R}^{n}\right)$ nor in $H^{k}\left(\mathbb{R}^{n}\right) \cap B M O\left(\mathbb{R}^{n}\right)$ and counterexemples are classically found in the form of series of needles. For $k=n / 2$, we have $H^{k}\left(\mathbb{R}^{n}\right) \cap$ $B M O\left(\mathbb{R}^{n}\right)=H^{k}\left(\mathbb{R}^{n}\right)$, whereas for $k>n / 2, H^{k}\left(\mathbb{R}^{n}\right)$ is included in the Wiener algebra $A\left(\mathbb{R}^{n}\right)$. We have introduced the natural simplifying assumption $u_{j}-u_{j, \infty} \in H^{k}\left(\mathbb{R}^{n}\right) \cap A\left(\mathbb{R}^{n}\right)$, which is sufficient for our applications, since for $k<n / 2, \mathcal{D}\left(\mathbb{R}^{n}\right)$ is dense in $H^{k}\left(\mathbb{R}^{n}\right) \cap A\left(\mathbb{R}^{n}\right)$ and $A\left(\mathbb{R}^{n}\right) \subset L^{\infty}\left(\mathbb{R}^{n}\right) \subset B M O\left(\mathbb{R}^{n}\right)$.

\subsection{Weighted products of derivatives}

We now estimate products of derivatives of temperature and velocity components rescaled by the proper temperature factors.

Théorème 3.7. Let $k \geq 1$ be an integer, $\bar{\theta}>0$ be positive, $1<p<\infty, T$ be such that $T \geq T_{\min }>0$ and $T-T_{\infty} \in H^{k}\left(\mathbb{R}^{n}\right) \cap A\left(\mathbb{R}^{n}\right)$ for some positive $T_{\infty}$. There exist positive constants $\delta(n, k, \bar{\theta}, p)$ and $c(n, k, p)$, only depending on $(n, k, \bar{\theta}, p)$ and $(n, k, p)$, respectively, such that if $\|\log T\|_{B M O}<\delta$, then for any real $\theta$ such that $|\theta| \leq \bar{\theta}$, any integer $l \geq 1$, and any multiindices $\alpha^{j}, 1 \leq j \leq l$, with $\left|\alpha^{j}\right| \geq 1,1 \leq j \leq l$, and $\sum_{1 \leq j \leq l}\left|\alpha^{j}\right|=k$, whenenver $T^{\theta / p}\left(\partial_{x}^{k} T\right) / T \in L^{p}\left(\mathbb{R}^{n}\right)$, we have the estimates

$$
\left\|T^{\frac{\theta}{p}} \prod_{1 \leq j \leq l}\left(\frac{\partial_{x}^{\alpha^{j}} T}{T}\right)\right\|_{L^{p}} \leq c\|\log T\|_{B M O}^{l-1}\left\|T^{\frac{\theta}{p}} \frac{\partial_{x}^{k} T}{T}\right\|_{L^{p}}
$$

Further assuming $\bar{v} \in H^{k}\left(\mathbb{R}^{n}\right) \cap A\left(\mathbb{R}^{n}\right),\|\log T\|_{B M O}+\|\bar{v} / \sqrt{T}\|_{L^{\infty}}<\delta(n, k, \bar{\theta}, p)$, and $T^{\theta / p}\left(\partial_{x}^{k} \bar{v}\right) / \sqrt{T} \in L^{p}\left(\mathbb{R}^{n}\right)$, we have for $0 \leq \bar{l} \leq l$

$$
\left\|T^{\frac{\theta}{p}} \prod_{1 \leq j \leq \bar{l}}\left(\frac{\partial_{x}^{\alpha^{j}} T}{T}\right) \prod_{\bar{l}+1 \leq j \leq l}\left(\frac{\partial_{x}^{\alpha^{j}} \bar{v}}{\sqrt{T}}\right)\right\|_{L^{p}} \leq c\left(\|\log T\|_{B M O}+\left\|\frac{\bar{v}}{\sqrt{T}}\right\|_{L^{\infty}}\right)^{l-1}\left(\left\|T^{\frac{\theta}{p}} \frac{\partial_{x}^{k} T}{T}\right\|_{L^{p}}+\left\|T^{\frac{\theta}{p}} \frac{\partial_{x}^{k} \bar{v}}{\sqrt{T}}\right\|_{L^{p}}\right),
$$


where, in the left hand member, with a slight abuse of notation, we have denoted by $\bar{v}$ any of its components $\bar{v}_{1}, \ldots, \bar{v}_{n}$. Moreover, the constants $\delta(n, k, \bar{\theta}, p)$ and $c(n, k, p)$, are independent of a change of scale in the coordinate system.

Proof. A detailed proof of the existence of $\delta(n, k, \bar{\theta}, p)$ and $c(n, k, p)$ is given in [11]. This proof essentially consists in applying inequality $(25)$ to $\tau=\log T$ and $\bar{w}=\bar{v} / \sqrt{T}$ and then estimating quantities like

$$
\left\|T^{\frac{\theta}{p}}\left(\prod_{1 \leq j \leq l}\left(\frac{\partial_{x}^{\alpha^{j}} T}{T}\right)-\prod_{1 \leq j \leq l} \partial_{x}^{\alpha^{j}} \tau\right)\right\|_{L^{p}},
$$

using again inequality (25). The scale invariance of $\delta(n, k, \bar{\theta}, p)$ and $c(n, k, p)$ is a direct consequence of the scaling properties of the $B M O$ and Lebesgue norms.

\section{Asymptotics of Higher order entropy estimates}

We investigate in this section higher order entropy asymptotic estimates for incompressible flows. We only investigate reduced unknowns and equations in this section and suppress hat accents from all symbols for the sake of notational simplicity.

\subsection{Rescaled governing equations}

We investigate the solutions of an incompressible model in reduced form (13)-(15) rewritten by suppressing hat accents

$$
\begin{gathered}
\partial_{x} \cdot v=0, \\
\partial_{t} v+\partial_{x} \cdot(v \otimes v)+\frac{1}{\epsilon_{\mathrm{M}}^{2}} \partial_{x} p-\frac{1}{\operatorname{Re}} \partial_{x} \cdot(\eta(T) d)=0, \\
\partial_{t}\left(c_{v} T\right)+\partial_{x} \cdot\left(c_{v} v T\right)-\frac{1}{\operatorname{Re}} \partial_{x} \cdot\left(\lambda(T) \partial_{x} T\right)=\frac{\epsilon_{\mathrm{M}}^{2}}{2 \operatorname{Re}} \eta(T) d: d,
\end{gathered}
$$

where $v$ is the velocity, $p$ the pressure, $d=\partial_{x} v+\partial_{x} v^{t}$ the strain rate tensor, $\eta(T)$ the viscosity, $T$ the absolute temperature and $\lambda(T)$ the thermal conductivity. All these quantities are dimensionless and we have assumed for the sake of simplicity that the internal energy is proportional to temperature $e=c_{v} T$ where $c_{v}$ is a constant. Note that, in comparison, all systems of partial differential equations considered in [10,11] did not contain any small parameter.

The relevant assumptions on the thermal conductivity $\lambda$ and the shear viscosity $\eta$ are derived from the kinetic theory of gases and by using the minimum principle for temperature as discussed in Section 2.5 [7,9, 11]. The reduced thermal conductivity and viscosity are $C^{\infty}(0, \infty)$ and such that there exist $\varkappa, \underline{\mathfrak{a}}>0, \overline{\mathfrak{a}}>0$, and $\overline{\mathfrak{a}}_{\sigma}>0$, for any integer $\sigma \geq 1$, so that

$$
\underline{\mathfrak{a}} T^{\varkappa} \leq \lambda / c_{v} \leq \overline{\mathfrak{a}} T^{\varkappa}, \quad \underline{\mathfrak{a}} T^{\varkappa} \leq \eta \leq \overline{\mathfrak{a}} T^{\varkappa}, \quad T^{\sigma}\left(\left|\partial_{T}^{\sigma} \lambda\right|+\left|\partial_{T}^{\sigma} \eta\right|\right) \leq \overline{\mathfrak{a}}_{\sigma} T^{\varkappa} .
$$

Kinetic theory suggets that $1 / 2 \leq \varkappa \leq 1$ but the situations where $0 \leq \varkappa<1 / 2$ or $\varkappa>1$ are still interesting to investigate from a mathematical point of view.

We consider the case of functions defined on $\mathbb{R}^{n}$ with $n \geq 2$, that are 'constant at infinity', and we only consider solutions such that

$$
v, T-T_{\infty} \in C\left(\left[0, t_{1}\right], H^{l}\right) \cap C^{1}\left(\left[0, t_{1}\right], H^{l-2}\right) \cap L^{2}\left(\left[0, t_{1}\right], H^{l+1}\right),
$$

where $l$ is an integer such that $l \geq[n / 2]+2$, that is, $l>n / 2+1$, and $t_{1}$ is some positive time. It can be shown that these solutions are smooth when the initial data is smooth [11]. Since the viscosity $\eta$ is not a constant, the momentum conservation equation is rewritten in the form $\partial_{t} v=\mathbb{P}\left(\partial_{x} \cdot(-v \otimes v+\eta(T) d)\right)$, where $\mathbb{P}$ is the Leray 
projector defined on $L^{2}\left(\mathbb{R}^{n}\right)^{n}$ by $\mathbb{P}=\mathbb{I}+R \otimes R$ where $R=\left(R_{1}, \ldots, R_{n}\right)^{t}$ and $R_{i}=(-\Delta)^{-1 / 2} \partial_{i}, 1 \leq i \leq n$, are the Riesz transforms. This is equivalent to defining the pressure from

$$
p=p_{0}+\epsilon_{\mathrm{M}}^{2} \sum_{1 \leq i, j \leq n} R_{i} R_{j}\left(v_{i} v_{j}-\eta d_{i j} / \mathrm{Re}\right),
$$

where $p_{0}$ is a constant pressure and we then have $p-p_{0} \in C\left(\left[0, t_{1}\right], H^{l}\right) \cap C^{1}\left(\left[0, t_{1}\right], H^{l-2}\right) \cap L^{2}\left(\left[0, t_{1}\right], H^{l+1}\right)$ and $\partial_{k} p=\epsilon_{\mathrm{M}}^{2} \sum_{1 \leq i, j \leq n} R_{k} R_{j}\left(v_{i} \partial_{i} v_{j}-2 \partial_{T} \eta \partial_{i} T \partial_{j} v_{i} / \mathrm{Re}\right)$.

Remarque 4.1. In the particular situation $\lambda=\mathfrak{a}_{\lambda} T^{\varkappa}$ and $\eta=\mathfrak{a}_{\eta} T^{\varkappa}$, where $\mathfrak{a}_{\lambda}$ and $\mathfrak{a}_{\eta}$ are constants, if $v(t, x)$ and $T(t, x)$ are a solution of the Navier-Stokes equations (29)-(31), then

$$
\xi v\left(\xi^{2(1-\varkappa)} t, \xi^{(1-2 \varkappa)} x\right), \quad \xi^{2} T\left(\xi^{2(1-\varkappa)} t, \xi^{(1-2 \varkappa)} x\right),
$$

are also a solution for any positive $\xi$.

\subsection{Higher order entropies}

Generalizing the expressions $(16)(24)$, we define the $(2 k)^{\text {th }}$ order rescaled kinetic entropy corrector $\gamma^{[k]}$ by

$$
\gamma^{[k]}=\epsilon_{\mathrm{K}}^{2 k}\left(\frac{\left|\partial^{k} T\right|^{2}}{T^{1+a_{k}}}+\epsilon_{\mathrm{M}}^{2} \frac{\left|\partial^{k} v\right|^{2}}{T^{a_{k}}}\right)=\epsilon_{\mathrm{K}}^{2 k}\left(\frac{\left|\partial^{k} T\right|^{2}}{T^{1+a_{k}}}+\frac{\left|\partial^{k} \bar{v}\right|^{2}}{T^{a_{k}}}\right),
$$

where $\left|\partial^{k} T\right|^{2}=\sum_{|\alpha|=k}(k ! / \alpha !)\left(\partial^{\alpha} T\right)^{2}$ and $\left|\partial^{k} v\right|^{2}=\sum_{1 \leq i \leq n}\left|\partial^{k} v_{i}\right|^{2}$, and where $k ! / \alpha !$ are the multinomial coefficients [4], keeping in mind that hat accents are now omitted. The renormalizing factors now involve arbitrary powers $a_{k}, k \geq 1$, of temperature. We can similarly introduce the modified rescaled kinetic entropy corrector $\tilde{\gamma}^{[k]}$ defined by

$$
\tilde{\gamma}^{[k]}=\frac{\epsilon_{\mathrm{K}}^{2 k}}{T^{a_{k}-1}}\left(\left|\partial^{k} \log T\right|^{2}+\epsilon_{\mathrm{M}}^{2}\left|\partial^{k}(v / \sqrt{T})\right|^{2}\right)=\frac{\epsilon_{\mathrm{K}}^{2 k}}{T^{a_{k}-1}}\left(\left|\partial^{k} \tau\right|^{2}+\epsilon_{\mathrm{M}}^{2}\left|\partial^{k} w\right|^{2}\right)=\frac{\epsilon_{\mathrm{K}}^{2 k}}{T^{a_{k}-1}}\left(\left|\partial^{k} \tau\right|^{2}+\left|\partial^{k} \bar{w}\right|^{2}\right),
$$

where $\tau=\log T, w=v / \sqrt{T}, \bar{w}=\epsilon_{\mathrm{M}} w=\bar{v} / \sqrt{T}$, and we will see that $\gamma^{[k]}$ and $\tilde{\gamma}^{[k]}$ have very similar properties.

Proposition 4.2. Let $k \geq 1$ be an integer and $(v, T)$ be a smooth solution of the incompressible Navier-Stokes equations (29)-(31). We then have the balance equation

$$
\partial_{t} \gamma^{[k]}+\partial_{x} \cdot\left(v \gamma^{[k]}\right)+\partial_{x} \cdot \varphi_{\gamma}^{[k]}+\pi_{\gamma}^{[k]}+\Sigma_{\gamma}^{[k]}+\omega_{\gamma}^{[k]}=0,
$$

where $\varphi_{\gamma}^{[k]}$ is a flux and $\pi_{\gamma}^{[k]}+\Sigma_{\gamma}^{[k]}+\omega_{\gamma}^{[k]}$ a source term. The term $\pi_{\gamma}^{[k]}$ is given by

$$
\pi_{\gamma}^{[k]}=\frac{\epsilon_{\mathrm{K}}^{2 k}}{\operatorname{Re}}\left(\frac{2 \lambda}{c_{v}} \frac{\left|\partial^{k+1} T\right|^{2}}{T^{1+a_{k}}}+2 \eta \epsilon_{\mathrm{M}}^{2} \frac{\left|\partial^{k+1} v\right|^{2}}{T^{a_{k}}}\right)=\frac{\epsilon_{\mathrm{K}}^{2 k}}{\operatorname{Re}}\left(\frac{2 \lambda}{c_{v}} \frac{\left|\partial^{k+1} T\right|^{2}}{T^{1+a_{k}}}+2 \eta \frac{\left|\partial^{k+1} \bar{v}\right|^{2}}{T^{a_{k}}}\right),
$$

in such a way that

$$
2 \underline{\mathfrak{a}} \gamma^{[k+1]} \leq \epsilon_{\mathrm{K}}^{2} \operatorname{Re} \pi_{\gamma}^{[k]} T^{-\left(a_{k+1}-a_{k}+\varkappa\right)} \leq 2 \overline{\mathfrak{a}} \gamma^{[k+1]} .
$$

The term $\Sigma_{\gamma}^{[k]}$ is given by

$$
\Sigma_{\gamma}^{[k]}=\sum_{\sigma \nu \mu} \frac{\epsilon_{\mathrm{K}}^{2 k}}{\operatorname{Re}}\left(c_{\sigma \nu \mu} \partial_{T}^{\sigma} \lambda+c_{\sigma \nu \mu}^{\prime} \partial_{T}^{\sigma} \eta\right) T^{\sigma-\varkappa} \Pi_{\nu}^{(k+1)} \Pi_{\mu}^{(k+1)}+\sum_{\sigma \nu \mu \mathcal{R}} \frac{\epsilon_{\mathrm{K}}^{2 k}}{\operatorname{Re}} c_{\sigma \nu \mu \mathcal{R}} \Pi_{\nu}^{(k+1)} \mathcal{R}\left(T^{\sigma-\varkappa} \partial_{T}^{\sigma} \eta \Pi_{\mu}^{(k+1)}\right),
$$


where the sums are over $0 \leq \sigma \leq k, \nu=\left(\nu_{\alpha}, \nu_{\alpha}^{\prime}\right)_{1 \leq|\alpha| \leq k+1}, \mu=\left(\mu_{\alpha}, \mu_{\alpha}^{\prime}\right)_{1 \leq|\alpha| \leq k+1}, \nu_{\alpha}, \nu_{\alpha}^{\prime}, \mu_{\alpha}, \mu_{\alpha}^{\prime} \in \mathbb{N}, \alpha \in \mathbb{N}^{n}$, and for $\mathcal{R}$ singular operator in the form $T^{-\theta} R_{i} R_{j} T^{\theta}$ with $\theta=\left(a_{k}+\varkappa\right) / 2$ and $1 \leq i, j \leq n$. The products $\Pi_{\nu}^{(k+1)}$ and $\Pi_{\mu}^{(k+1)}$ are given by

$$
\Pi_{\nu}^{(k+1)}=T^{\left(1-a_{k}+\varkappa\right) / 2} \prod_{1 \leq|\alpha| \leq k+1}\left(\frac{\partial^{\alpha} T}{T}\right)^{\nu_{\alpha}}\left(\frac{\partial^{\alpha}\left(\epsilon_{\mathrm{M}} v\right)}{\sqrt{T}}\right)^{\nu_{\alpha}^{\prime}}
$$

where $v$ denotes - with a sligth abuse of notation - any of its components $v_{1}, \ldots, v_{n}$, and $\mu$ and $\nu$ are such that $\sum_{1 \leq|\alpha| \leq k+1}|\alpha|\left(\nu_{\alpha}+\nu_{\alpha}^{\prime}\right)=\sum_{1 \leq|\alpha| \leq k+1}|\alpha|\left(\mu_{\alpha}+\mu_{\alpha}^{\prime}\right)=k+1, \sum_{|\alpha|=k+1}\left(\nu_{\alpha}+\nu_{\alpha}^{\prime}+\mu_{\alpha}+\mu_{\alpha}^{\prime}\right) \leq 1$, in such a way that there is at most one derivative of order $k+1$ in the product $\Pi_{\nu}^{(k+1)} \Pi_{\mu}^{(k+1)}$, and the coefficients $c_{\sigma \nu \mu}$, $c_{\sigma \nu \mu}^{\prime}$, et $c_{\sigma \nu \mu \mathcal{R}}$ are constants. Finally, the term $\omega_{\gamma}^{[k]}$ can be written

$$
\omega_{\gamma}^{[k]} T^{-\left(1-2 \varkappa+a_{k-1}-a_{k}\right) / 2}=\sum_{\nu \mu} \frac{\epsilon_{\mathrm{K}}^{2 k-1}}{\operatorname{Re}} c_{\nu \mu} \Pi_{\nu}^{(k)} \Pi_{\mu}^{(k+1)}+\sum_{\nu \mu \mathcal{R}} \frac{\epsilon_{\mathrm{K}}^{2 k-1}}{\operatorname{Re}} c_{\nu \mu \mathcal{R}} \Pi_{\nu}^{(k)} \mathcal{R}\left(\Pi_{\mu}^{(k+1)}\right),
$$

where we sum over $\sum_{1 \leq|\alpha| \leq k}|\alpha|\left(\nu_{\alpha}+\nu_{\alpha}^{\prime}\right)=k, \sum_{1 \leq|\alpha| \leq k}|\alpha|\left(\mu_{\alpha}+\mu_{\alpha}^{\prime}\right)=k+1$, so that in particular $\sum_{|\alpha|=k+1}\left(\mu_{\alpha}+\mu_{\alpha}^{\prime}\right)=0$ and there are always two factors in the product $\Pi_{\mu}^{(k+1)}$, and where $\mathcal{R}$ is a singular operator in the form $T^{-\theta} R_{i} R_{j} T^{\theta}$ with $\theta=\left(1+a_{k}-\varkappa\right) / 2$ and $1 \leq i, j \leq n$.

Proof. The proof is lenghty but does not present serious difficulties other than notational and is similar to the proofs given in [11] for unscaled equations.

Remarque 4.3. Note that the velocity $\bar{v}=\epsilon_{\mathrm{M}} v$ naturally appears in the multilinear products (41). A similar balance equation can also be established for the rescaled modified higher order entropy $\tilde{\gamma}^{[k]}(36)[11]$.

Remarque 4.4. Replacing $\partial_{x} v$ by $d$ in the definition of $\gamma^{[k]}$ would yield

$$
\breve{\gamma}^{[k]}=\epsilon_{\mathrm{K}}^{2 k}\left(\frac{\left|\partial_{x}^{k} T\right|^{2}}{T^{1+a_{k}}}+\epsilon_{\mathrm{M}}^{2} \frac{\left|\partial_{x}^{k-1} d\right|^{2}}{T^{a_{k}}}\right)=\epsilon_{\mathrm{K}}^{2 k}\left(\frac{\left|\partial_{x}^{k} T\right|^{2}}{T^{1+a_{k}}}+\frac{\left|\partial_{x}^{k-1}\left(\partial_{x} \bar{v}+\partial_{x} \bar{v}^{t}\right)\right|^{2}}{T^{a_{k}}}\right),
$$

which is similar for $k=1$ to the quantity $-S^{(2)}$ introduced in Section 1.2. However, the definitions (6), (7), (35), and $\breve{\gamma}^{[k]}$ are equivalent for $k \geq 2$ since $2 \partial_{j} \partial_{l} v_{i}=\partial_{l} d_{i j}+\partial_{j} d_{i l}-\partial_{i} d_{j l}$ for any $v \in H^{1}$ and any indices $(i, j, l)$ and yield similar results for $k=1$ since for any $v \in H^{1}$ and any index pair $(i, j)$ we have [21]

$$
2 \partial_{j} v_{i}=d_{i j}-\sum_{1 \leq l \leq n} R_{l} R_{j} d_{l i}+\sum_{1 \leq l \leq n} R_{l} R_{i} d_{l j}
$$

where $R_{i}=(-\Delta)^{-1 / 2} \partial_{i}$ are the Riesz transforms, $1 \leq i \leq n$, keeping in mind that the operators $T^{\theta} R_{i} T^{-\theta}$ are continuous for $\|\log T\|_{B M O}$ small enough.

\subsection{A priori estimates}

We now introduce the quantity

$$
\chi=\|\log T\|_{B M O}+\epsilon_{\mathrm{M}}\|v / \sqrt{T}\|_{L^{\infty}}=\|\log T\|_{B M O}+\|\bar{v} / \sqrt{T}\|_{L^{\infty}}=\|\tau\|_{B M O}+\|\bar{w}\|_{L^{\infty}},
$$

and we want to estimate $\int_{\mathbb{R}^{n}}\left|\Sigma_{\gamma}^{[k]}\right| d x$ with $\int_{\mathbb{R}^{n}} \pi_{\gamma}^{[k]} d x$ when $\chi$ is small egough, using the weighted inequalities established in the Section 3. This quantity $\chi$ is invariant by the change of scales of Remark 4.1 and involve the natural variables $\tau=\log T$ and $\bar{w}=\epsilon_{\mathrm{M}} v / \sqrt{T}$ associated with Maxwellian distributions. A constraint in the form $\chi<\delta$ can also be interpreted heuristically as a constraint on the Mach number since formally $\chi=\mathcal{O}\left(\epsilon_{\mathrm{M}}\right)$. 
Proposition 4.5. Let $k \geq 1$ be an integer and $(v, T)$ be a smooth solution of the incompressible Navier-Stokes equations (29)-(31). There exists positive constants $\delta(k, n)$ and $c(k, n)$-independent of $\epsilon_{\mathrm{K}}$ and $\epsilon_{\mathrm{M}}$-such that for $\chi<\delta$ we have

$$
\int_{\mathbb{R}^{n}}\left|\Sigma_{\gamma}^{[k]}\right| d x \leq c \chi \int_{\mathbb{R}^{n}} \pi_{\gamma}^{[k]} d x
$$

Proof. The multiplicative factors $\epsilon_{\mathrm{K}}^{2 k}$ are identical on both sides and do not introduce difficulties. From the expressions (40) for $\Sigma_{\gamma}^{[k]}$, since the quantities $T^{\sigma-\varkappa} \partial_{T}^{\sigma} \lambda$ and $T^{\sigma-\varkappa} \partial_{T}^{\sigma} \eta$ are uniformly bounded from assumptions (23) (32), and since the operators $T^{\theta} R_{i} R_{j} T^{-\theta}$ are continuous over $L^{2}$ for $\|\log T\|_{B M O}$ small enough, we only have to estimate the $L^{2}$ norm of the products $\Pi_{\nu}^{(k+1)}$ and $\Pi_{\mu}^{(k+1)}$. However, using the multilinear estimates of Theorem 3.7 with $p=2$, we obtain when $\|\log T\|_{B M O}+\|\bar{v} / \sqrt{T}\|_{L^{\infty}}<\delta(k, n)$ the weighted inequalities

$$
\left\|\Pi_{\nu}^{(k+1)}\right\|_{L^{2}} \leq c \chi^{N_{\nu}-1}\left(\left\|T^{\frac{\theta}{2}} \frac{\partial^{k+1} T}{T}\right\|_{L^{2}}+\left\|T^{\frac{\theta}{2}} \frac{\partial^{k+1} \bar{v}}{\sqrt{T}}\right\|_{L^{2}}\right)
$$

where $\theta=1-a_{k}+\varkappa, c=c(k, n)$, and $N_{\nu}=\sum_{1 \leq|\alpha| \leq k+1}\left(\nu_{\alpha}+\nu_{\alpha}^{\prime}\right)$. As a consequence, inserting the $\epsilon_{\mathrm{K}}^{2 k}$ factors, we have

$$
\epsilon_{\mathrm{K}}^{2 k}\left\|\Pi_{\nu}^{(k+1)}\right\|_{L^{2}}\left\|\Pi_{\mu}^{(k+1)}\right\|_{L^{2}} \leq c \chi^{N_{\nu}+N_{\mu}-2} \int_{\mathbb{R}^{n}} \pi_{\gamma}^{[k]} d x
$$

and the proof is complete from $N_{\nu}+N_{\mu}-2=\sum_{1 \leq|\alpha| \leq k+1}\left(\nu_{\alpha}+\nu_{\alpha}^{\prime}+\mu_{\alpha}+\mu_{\alpha}^{\prime}\right)-2 \geq 1$ since at least one of the products $\Pi_{\nu}^{(k+1)}$ or $\Pi_{\mu}^{(k+1)}$ is split into two or more derivative factors.

These estimates imply in particular that $(1-c \chi) \int_{\mathbb{R}^{n}} \pi_{\gamma}^{[k]} d x \leq \int_{\mathbb{R}^{n}}\left(\pi_{\gamma}^{[k]}+\Sigma_{\gamma}^{[k]}\right) d x \leq(1+c \chi) \int_{\mathbb{R}^{n}} \pi_{\gamma}^{[k]} d x$ as soon as $\chi$ is small enough, so that

$$
\partial_{t} \int_{\mathbb{R}^{n}} \gamma^{[k]} d x+(1-c \chi) \int_{\mathbb{R}^{n}} \pi_{\gamma}^{[k]} d x \leq \int_{\mathbb{R}^{n}}\left|\omega_{\gamma}^{[k]}\right| d x
$$

One can also establish, by using the same type of techniques, that $\int_{\mathbb{R}^{n}}\left|\gamma^{[k]}-\tilde{\gamma}^{[k]}\right| d x \leq c \chi \int_{\mathbb{R}^{n}} \gamma^{[k]} d x$ so that $\int_{\mathbb{R}^{n}} \gamma^{[k]} d x$ and $\int_{\mathbb{R}^{n}} \tilde{\gamma}^{[k]} d x$ are asymptotically equivalent as $\chi \rightarrow 0$. We now estimate the contributions arising from convective terms.

Proposition 4.6. Let $k \geq 1$ be an integer and $(v, T)$ be a smooth solution of the incompressible Navier-Stokes equations (29)-(31). There exists positive constants $\delta(k, n)$ and $c(k, n)$-independent of $\epsilon_{\mathrm{K}}$ and $\epsilon_{\mathrm{M}}$-such that for $\chi<\delta$ we have

$$
\int_{\mathbb{R}^{n}}\left|\omega_{\gamma}^{[k]}\right| d x \leq c \chi \sup _{\mathbb{R}^{n}}\left\{T^{\left(1-2 \varkappa+a_{k-1}-a_{k}\right) / 2}\right\}\left(\int_{\mathbb{R}^{n}} \pi_{\gamma}^{[k-1]} d x\right)^{\frac{1}{2}}\left(\int_{\mathbb{R}^{n}} \pi_{\gamma}^{[k]} d x\right)^{\frac{1}{2}}
$$

Proof. From (42) and the continuity of the operators $T^{\theta} R_{i} R_{j} T^{-\theta}$ for $\|\log T\|_{B M O}$ small enough, we deduce after some algebra that

$$
\int_{\mathbb{R}^{n}}\left|\omega_{\gamma}^{[k]}\right| d x \leq c \sup _{\mathbb{R}^{n}}\left\{T^{\left(1-2 \varkappa+a_{k-1}-a_{k}\right) / 2}\right\} \epsilon_{\mathrm{K}}^{2 k-1}\left\|\Pi_{\nu}^{(k)}\right\|_{L^{2}}\left\|\Pi_{\mu}^{(k+1)}\right\|_{L^{2}},
$$

and the estimate (46) is a direct consequence of the inequalities established in the proof of Proposition 4.5 since there are at least two derivatives factors in the product $\Pi_{\mu}^{(k+1)}$.

Corollaire 4.7. Let $k \geq 1$ be an integer and $(v, T)$ be a smooth solution of the incompressible Navier-Stokes equations (29)-(31). There exists positive constants $\delta_{\chi}(k, n)$ and $c(k, n)$-independent of $\epsilon_{\mathrm{K}}$ and $\epsilon_{\mathrm{M}}$-such that 
for $\chi<\delta_{\chi}$ we have

$$
\partial_{t} \int_{\mathbb{R}^{n}} \gamma^{[k]} d x+\frac{\underline{\mathfrak{a}}}{\epsilon_{\mathrm{K}}^{2} \operatorname{Re}} \int_{\mathbb{R}^{n}} T^{a_{k+1}-a_{k}+\varkappa} \gamma^{[k+1]} d x \leq \frac{c \chi^{2}}{\epsilon_{\mathrm{K}}^{2} \operatorname{Re}} \sup _{\mathbb{R}^{n}} T^{\left(1-2 \varkappa+a_{k-1}-a_{k}\right)} \int_{\mathbb{R}^{n}} T^{a_{k}-a_{k-1}+\varkappa} \gamma^{[k]} d x
$$

with similar results for the modified entropies

$$
\partial_{t} \int_{\mathbb{R}^{n}} \tilde{\gamma}^{[k]} d x+\frac{\underline{\mathfrak{a}}}{\epsilon_{\mathrm{K}}^{2} \operatorname{Re}} \int_{\mathbb{R}^{n}} T^{a_{k+1}-a_{k}+\varkappa} \tilde{\gamma}^{[k+1]} d x \leq \frac{c \chi^{2}}{\epsilon_{\mathrm{K}}^{2} \operatorname{Re}} \sup _{\mathbb{R}^{n}} T^{\left(1-2 \varkappa+a_{k-1}-a_{k}\right)} \int_{\mathbb{R}^{n}} T^{a_{k}-a_{k-1}+\varkappa} \tilde{\gamma}^{[k]} d x .
$$

Proof. The first inequality (47) is a consequence of Propositions 4.5, 4.6 and $2 z \leq \varepsilon+z^{2} / \varepsilon$. The proof of the second inequality (48) is similar and the details are omitted.

\subsection{Zeroth order estimates}

In order to recast the zeroth order entropy balance into a convenient form, we now define $\gamma^{[0]}=\tilde{\gamma}^{[0]}$, for $0<a_{0} \leq 1$, by

$$
\gamma^{[0]}=\tilde{\gamma}^{[0]}= \begin{cases}\frac{T-T_{\infty}}{T_{\infty}}-\log \left(\frac{T}{T_{\infty}}\right)+\frac{1}{2} \epsilon_{\mathrm{M}}^{2} \frac{v^{2}}{c_{v} T_{\infty}}, & \text { if } a_{0}=1, \\ \frac{T-T_{\infty}}{T_{\infty}^{a_{0}}}-\frac{T^{1-a_{0}}-T_{\infty}^{1-a_{0}}}{1-a_{0}}+\frac{1}{2} \epsilon_{\mathrm{M}}^{2} \frac{v^{2}}{c_{v} T_{\infty}^{a_{0}}}, & \text { if } 0<a_{0}<1 .\end{cases}
$$

Proposition 4.8. Let $0<a_{0} \leq 1$ and let $\gamma^{[0]}$ be given by (49). Then $\gamma^{[0]} \geq 0$ and there exists positive constants $\delta_{0}>0$ and $\underline{b}_{0}^{\prime}$ such that for $\chi<\delta_{0}$ small enough

$$
\partial_{t} \int_{\mathbb{R}^{n}} \gamma^{[0]} d x+\underline{b}_{0}^{\prime} \int_{\mathbb{R}^{n}} \pi_{\gamma}^{[0]} d x \leq 0
$$

where we define from (38)

$$
\pi_{\gamma}^{[0]}=\frac{1}{\operatorname{Re}}\left(\frac{2 \lambda}{c_{v}} \frac{\left|\partial_{x}^{1} T\right|^{2}}{T^{1+a_{0}}}+2 \eta \epsilon_{\mathrm{M}}^{2} \frac{\left|\partial_{x}^{1} v\right|^{2}}{T^{a_{0}}}\right)=\frac{1}{\operatorname{Re}}\left(\frac{2 \lambda}{c_{v}} \frac{\left|\partial_{x}^{1} T\right|^{2}}{T^{1+a_{0}}}+2 \eta \frac{\left|\partial_{x}^{1} \bar{v}\right|^{2}}{T^{a_{0}}}\right) .
$$

Equivalently, there exists a positive constant $\underline{b}_{0}$ such that for $\chi<\delta_{0}$ we have

$$
\partial_{t} \int_{\mathbb{R}^{n}} \gamma^{[0]} d x+\frac{2 \underline{b}_{0}}{\epsilon_{\mathrm{K}}^{2} \operatorname{Re}} \int_{\mathbb{R}^{n}} T^{\varkappa+a_{1}-a_{0}} \gamma^{[1]} d x \leq 0,
$$

with a similar result for the modified entropies

$$
\partial_{t} \int_{\mathbb{R}^{n}} \tilde{\gamma}^{[0]} d x+\frac{2 \underline{b}_{0}}{\epsilon_{\mathrm{K}}^{2} \operatorname{Re}} \int_{\mathbb{R}^{n}} T^{\varkappa+a_{1}-a_{0}} \tilde{\gamma}^{[1]} d x \leq 0 .
$$

Proof. We only consider the case $a_{0}=1$ since the case $0<a_{0}<1$ is similar. It is first easily established that the temperature part of $\gamma^{[0]}$ is nonnegative so that $\gamma^{[0]} \geq 0$. Dividing the temperature equation by $T$ and integrating over $\mathbb{R}^{n}$ we obtain after some algebra

$$
-\partial_{t} \int_{\mathbb{R}^{n}} \log \left(\frac{T}{T_{\infty}}\right) d x+\frac{1}{\operatorname{Re} c_{v}} \int_{\mathbb{R}^{n}} \frac{\lambda\left|\partial_{x} T\right|^{2}}{T^{2}} d x+\frac{\epsilon_{\mathrm{M}}^{2}}{2 \operatorname{Re} c_{v}} \int_{\mathbb{R}^{n}} \frac{\eta|d|^{2}}{T} d x=0
$$


On the other hand, dividing the total energy conservation equation by $T_{\infty}$ and integrating over $\mathbb{R}^{n}$ we obtain

$$
\partial_{t} \int_{\mathbb{R}^{n}}\left(\frac{T-T_{\infty}}{T_{\infty}}+\frac{1}{2} \frac{\epsilon_{\mathrm{M}}^{2} v^{2}}{c_{v} T_{\infty}}\right) d x=0
$$

Finally, from the relations between $\partial_{x} v$ and $d$ of Remark 4.4, we obtain $\int_{\mathbb{R}^{n}} \frac{\left|\partial_{x} v\right|^{2}}{T^{1-\varkappa}} d x \leq c \int_{\mathbb{R}^{n}} \frac{|d|^{2}}{T^{1-\varkappa}} d x$ for $\|\log T\|_{B M O}$ small enough and combining these estimates completes the proof of (50) and (51). Inequality (52) is then a consequence of (51) and of $\partial_{x} \bar{w}=\partial_{x} \bar{v} / \sqrt{T}-\frac{1}{2} \bar{w} \otimes \partial_{x} \tau$ [11].

\subsection{Higher order entropic inequalities}

We define the $(2 k)^{\text {th }}$ order kinetic entropy estimators by $\Gamma^{[k]}=\gamma^{[0]}+\cdots+\gamma^{[k]}$ and $\widetilde{\Gamma}^{[k]}=\tilde{\gamma}^{[0]}+\cdots+\tilde{\gamma}^{[k]}$, for $k \geq 0$, so that

$$
\begin{gathered}
\Gamma^{[k]}=\gamma^{[0]}+\sum_{1 \leq l \leq k} \epsilon_{\mathrm{K}}^{2 l}\left(\frac{\left|\partial_{x}^{l} T\right|^{2}}{T^{1+a_{l}}}+\epsilon_{\mathrm{M}}^{2} \frac{\left|\partial_{x}^{l} v\right|^{2}}{T^{a_{l}}}\right), \\
\widetilde{\Gamma}^{[k]}=\tilde{\gamma}^{[0]}+\sum_{1 \leq l \leq k} \frac{\epsilon_{\mathrm{K}}^{2 l}}{T^{a_{l}-1}}\left(\left|\partial_{x}^{l} \tau\right|^{2}+\epsilon_{\mathrm{M}}^{2}\left|\partial_{x}^{l} w\right|^{2}\right),
\end{gathered}
$$

and we have to establish that these kinetic entropy estimators obey entropic principles for the solutions of the incompressible fluid model (29)-(31). From a terminology point of view, strictly speaking, we should term $\gamma^{[k]}$ and $\tilde{\gamma}^{[k]}$ "mathematical $(2 k)^{\text {th }}$ order partial entropies" or " $(2 k)^{\text {th }}$ order kinetic entropy majorizing correctors" or " $(2 k)^{\text {th }}$ order kinetic entropy deviation estimators" and $\Gamma^{[k]}$ and $\widetilde{\Gamma}^{[k]}$ "mathematical $(2 k)^{\text {th }}$ order entropies", or " $(2 k)^{\text {th }}$ order kinetic entropy estimators". However, we informally term $\gamma^{[k]}, \tilde{\gamma}^{[k]}, \Gamma^{[k]}$ and $\widetilde{\Gamma}^{[k]}$ "mathematical $(2 k)^{\text {th }}$ order entropies" or simply "higher order entropies".

In order to combine the estimates obtained in the previous sections, we need to specify the scale of temperature weights $a_{k}, k \geq 0$, used to renormalize the successive derivatives of $T$ and of $\epsilon_{\mathrm{M}} v=\bar{v}$. A first possibility is to impose that all $\sup _{\mathbb{R}^{n}} T^{z}$ factors of the convective terms estimates of Proposition 4.6 and of Corollary 4.7 disappear by letting $z=0$, that is, by letting $1-2 \varkappa+a_{k-1}-a_{k}=0, k \geq 1$, in such a way that

$$
a_{k}=a_{0}+k(1-2 \varkappa), \quad k \geq 0 .
$$

This scale fulfills the natural requirement that estimates for $\gamma^{[k-1]}$ and $\pi_{\gamma}^{[k-1]}$ and the conditional entropicity property (45) yield estimates for $\gamma^{[k]}$ and $\pi_{\gamma}^{[k]}$. This scale of temperature weights also corresponds to the scale given by the kinetic theory of gases with (16) (24) since the factor $(\eta / \rho \sqrt{r T})^{2 k}=\left(\eta^{2} / \rho^{2} r T\right)^{k}$ yields the temperature exponent $k(1-2 \varkappa)$ from assumptions (32) and incompressibility. This scale of temperature weights is also important for compressible models [12]. Therefore, this scale $a_{k}=a_{0}+k(1-2 \varkappa), k \geq 0$, can be termed the natural scale of temperature weights. It is interesting to note that with this scale, $a_{k}$ is decreasing with $k$ for physical values of $\varkappa$, that is, for values such that $\varkappa \geq 1 / 2$. On the other hand, $a_{k}$ is increasing with $k$ for unphysical values of $\varkappa$, that is, for values such that $0 \leq \varkappa<1 / 2$. This means in particular that, in the unphysical situation $0 \leq \varkappa<1 / 2$, larger powers of $T$ are needed in order to renormalize higher derivatives so that only the situation $\varkappa \geq 1 / 2$ seems to be interesting from a practical point of view.

On the other hand, one can also impose that the temperature weights are all equal

$$
a_{k}=a_{0}, \quad k \geq 0,
$$

and this scale of temperature weights is termed the uniform scale. It is important to note that the $\sup _{\mathbb{R}^{n}} T^{z}$ factors of the right members of (46) in Proposition 4.6 cannot be majorized in terms of the solution derivatives since $T_{\infty}>0$. As a consequence, taking into account the natural lower bound for temperature in terms of initial data $T \geq T_{\min }>0$, controling these $\sup _{\mathbb{R}^{n}} T^{z}$ factors require to have negative $z$ exponents in (46). Therefore, 
we must have $1-2 \varkappa+a_{k-1}-a_{k} \leq 0, k \geq 1$, and thus $a_{0}+k(1-2 \varkappa) \leq a_{k}$, for $k \geq 0$, and the natural scale of temperature weights appears to be a lower bound among all the useful scales. In particular, selecting a uniform scale requires that $k(1-2 \varkappa) \leq$ Cte, so that we must have $\varkappa \geq 1 / 2$. In other words, the transport coefficients have to follow the temperature dependence given by the kinetic theory in order to use a uniform scale. With this scale, higher order entropy estimates directly yield estimates of higher order derivatives of $\log T$ and $\bar{v} / \sqrt{T}$.

Théorème 4.9. Let $k \geq 1$ be an integer and $(v, T)$ be a smooth solution of the incompressible Navier-Stokes equations (29)-(31). Assume that $\varkappa \geq 1 / 2, a_{l}=a_{0}, l \geq 0, T_{\min } \leq T$, and let $k \geq 1$ be fixed. There exists positive constants $\underline{b}=\min \left(\underline{b}_{0}, \underline{\mathfrak{a}}\right)$ and $\delta_{\mathrm{U}}\left(k, n, T_{\min }\right)$ such that for $\chi<\delta_{\mathrm{U}}$ we have the estimates

$$
\partial_{t} \int_{\mathbb{R}^{n}}\left(\gamma^{[0]}+\cdots+\gamma^{[k]}\right) d x+\frac{\underline{b}}{\epsilon_{\mathrm{K}}^{2} \operatorname{Re}} \int_{\mathbb{R}^{n}} T^{\varkappa}\left(\gamma^{[1]}+\cdots+\gamma^{[k+1]}\right) d x \leq 0,
$$

with similar results for the modified higher order entropies

$$
\partial_{t} \int_{\mathbb{R}^{n}}\left(\tilde{\gamma}^{[0]}+\cdots+\tilde{\gamma}^{[k]}\right) d x+\frac{\underline{b}}{\epsilon_{\mathrm{K}}^{2} \operatorname{Re}} \int_{\mathbb{R}^{n}} T^{\varkappa}\left(\tilde{\gamma}^{[1]}+\cdots+\tilde{\gamma}^{[k+1]}\right) d x \leq 0 .
$$

Proof. The inequality (55) is a consequence of the estimates of Propositions 4.5, 4.6 and 4.8, and the proof of (56) is similar.

Theorem 4.9 shows that the $(2 k)^{\text {th }}$ order kinetic entropy estimators $\Gamma^{[k]}=\gamma^{[0]}+\gamma^{[1]}+\cdots+\gamma^{[k]}$ and $\widetilde{\Gamma}^{[k]}=$ $\tilde{\gamma}^{[0]}+\tilde{\gamma}^{[1]}+\cdots+\tilde{\gamma}^{[k]}$ effectively obey entropic principles. These higher order entropic inequalities yield new a priori estimates as soon as the quantity $\chi=\|\log T\|_{B M O}+\epsilon_{\mathrm{M}}\|v / \sqrt{T}\|_{L^{\infty}}$ is small enough. This quantity is small when the Mach number is small since formally $\chi=\mathcal{O}\left(\epsilon_{\mathrm{M}}\right)$. The estimates (56) are especially useful in the situation of logarithmic scaling $a_{k}=1, k \geq 0$, since they directly yield estimates for derivatives of $\tau=\log T$ and $\bar{w}=\epsilon_{\mathrm{M}} v / \sqrt{T}$ in the form

$$
\partial_{t} \int_{\mathbb{R}^{n}}\left(\tilde{\gamma}^{[0]}+\sum_{1 \leq l \leq k} \epsilon_{\mathrm{K}}^{2 l}\left(\left|\partial_{x}^{l} \tau\right|^{2}+\left|\partial_{x}^{l} \bar{w}\right|^{2}\right)\right) d x+\frac{\underline{b}}{\operatorname{Re}} \int_{\mathbb{R}^{n}} T^{\varkappa} \sum_{1 \leq l \leq k+1} \epsilon_{\mathrm{K}}^{2(l-1)}\left(\left|\partial_{x}^{l} \tau\right|^{2}+\left|\partial_{x}^{l} \bar{w}\right|^{2}\right) d x \leq 0 .
$$

Furthermore, taking into account $\bar{w}^{2} \leq \bar{v}^{2} / T_{\min }$ and the inequality

$$
\frac{T_{\min }}{2 T_{\infty}}\left|\tau-\tau_{\infty}\right|^{2} \leq \exp \left(\tau-\tau_{\infty}\right)-1-\left(\tau-\tau_{\infty}\right),
$$

valid for $\tau_{\min }=\log T_{\min } \leq \tau$, where $\tau_{\infty}=\log T_{\infty}$ and $T_{\min } \leq T_{\infty}$, we deduce that

$$
\sum_{0 \leq l \leq k} \epsilon_{\mathrm{K}}^{2 l}\left(\left|\partial_{x}^{l}\left(\tau-\tau_{\infty}\right)\right|^{2}+\left|\partial_{x}^{l} \bar{w}\right|^{2}\right)=\sum_{0 \leq l \leq k}\left(\left|\partial_{\bar{x}}^{l}\left(\tau-\tau_{\infty}\right)\right|^{2}+\left|\partial_{\bar{x}}^{l} \bar{w}\right|^{2}\right) \leq c\left(T_{\min }\right) \widetilde{\Gamma}^{[k]},
$$

for some constant $c\left(T_{\text {min }}\right)$ where $x=\epsilon_{\mathrm{K}} \bar{x}$. In other words, so that $\epsilon_{\mathrm{K}}^{-n} \int_{\mathbb{R}^{n}} \widetilde{\Gamma}^{[k]} d x=\int_{\mathbb{R}^{n}} \widetilde{\Gamma}^{[k]} d \bar{x}$ essentially represents a Sobolev norm of $\overline{\mathrm{W}}-\overline{\mathrm{W}}_{\infty}=\left(\bar{w}, \tau-\tau_{\infty}\right)$ in molecular coordinates $\bar{x}$.

\section{Application to ASymptotic stability}

As an example of application of higher order entropy estimates we investigate in this section global existence of solutions for small Mach and Knudsen numbers. We assume throughout this section that the scale of temperature weights is uniform with $a_{k}=1, k \geq 0$, and that the transport coefficients $\lambda$ and $\eta$ satisfy assumptions (23) (32) with $\varkappa \geq 1 / 2$. We also assume that the initial state is such that $\tau_{0}-\tau_{\infty}=\mathcal{O}\left(\epsilon_{\mathrm{M}}\right)$ and $\bar{w}_{0}=\mathcal{O}\left(\epsilon_{\mathrm{M}}\right)$. 
We first use the rescaled equations in molecular coordinates for convenience and strenghen an existence result obtained in $[10,11]$. We indeed investigate a priori bounds of kinetic entropic correctors in the form of powers of the Knudsen number. These estimates are then combined with the local existence results of [10,11] in order to establish existence of a global solution. The derivatives of the corresponding solutions properly scale as powers of the Knudsen number and these results are then mapped back to the physical variables.

\subsection{A stable domain}

We denote by $\overline{\mathrm{W}}$ the combined unknown $\overline{\mathrm{W}}=(\bar{w}, \tau)$, where $\tau=\log T$ and $\bar{w}=\epsilon_{\mathrm{M}} w=\epsilon_{\mathrm{M}} v / \sqrt{T}$, and we also define $\overline{\mathrm{V}}=(\bar{v}, T)$. We denote accordingly by $\overline{\mathrm{W}}_{\infty}$ the equilibrium point $\overline{\mathrm{W}}_{\infty}=\left(0, \log T_{\infty}\right)$ with $\bar{w}_{\infty}=0$ and $T_{\infty}>0$. We denote by $\mathcal{O}_{\overline{\mathrm{w}}}=\mathbb{R}^{n+1}$ the natural domain for the variable $\overline{\mathrm{W}}$, where $n \geq 2$. We use the molecular coordinates $(\bar{t}, \bar{x})$, where $\bar{t}=t / \epsilon_{\mathrm{M}} \epsilon_{\mathrm{K}}=t / \epsilon_{\mathrm{K}}^{2}$ Re and $\bar{x}=x / \epsilon_{\mathrm{K}}$, and the corresponding rescaled equations presented in Section 2.4. We will frequently commit the small abuse of notation of denoting by the same letter a function of $\bar{x}$ and the corresponding function of the macroscopic variable $x$ for the sake of notational simplicity.

If $\bar{\phi}$ is a smooth function of $\bar{x}$, then $\int_{\mathbb{R}^{n}}\left|\partial_{\bar{x}}^{k} \bar{\phi}\right|^{2} d \bar{x}=\epsilon_{\mathrm{K}}^{2 k-n} \int_{\mathbb{R}^{n}}\left|\partial_{x}^{k} \bar{\phi}\right|^{2} d x$, and further assuming $\bar{\phi}=\epsilon_{\mathrm{M}} \phi$ we have

$$
\int_{\mathbb{R}^{n}}\left|\partial_{\bar{x}}^{k} \bar{\phi}\right|^{2} d \bar{x}=\epsilon_{\mathrm{K}}^{2 k+2-n} \operatorname{Re}^{2} \int_{\mathbb{R}^{n}}\left|\partial_{x}^{k} \phi\right|^{2} d x
$$

so that $\int_{\mathbb{R}^{n}}\left|\partial_{\bar{x}}^{k} \bar{\phi}\right|^{2} d \bar{x}$ is of the order $\epsilon_{\mathrm{K}}^{2 k+2-n}$ if the integral $\operatorname{Re}^{2} \int_{\mathbb{R}^{n}}\left|\partial_{x}^{k} \phi\right|^{2} d x$ is of order unity. We investigate in the following a priori estimates of higher order entropy corrector integrals $\int_{\mathbb{R}^{n}} \tilde{\gamma}^{[k]} d \bar{x}$ with such a scaling law $\epsilon_{\mathrm{K}}^{2 k+2-n}$. Note that composite quantities like $\widetilde{\Gamma}^{[k]}$ cannot be used anymore in order to establish such a scaling law $\epsilon_{\mathrm{K}}^{2 k+2-n}$ for $\tilde{\gamma}^{[k]}$ or $\gamma^{[k]}$ since there is a different exponent $2 k+2-n$ for each derivative index $k$.

A fundamental difficulty associated with molecular coordinates is that space integrals $\int_{\mathbb{R}^{n}} \phi d \bar{x}$ are $\mathcal{O}\left(\epsilon_{\mathrm{K}}^{-n}\right)$ with respect to the corresponding space integrals $\int_{\mathbb{R}^{n}} \phi d x$ in macroscopic coordinates $x$. These $\epsilon_{\mathrm{K}}^{-n}$ factors introduce extra difficulties for controlling $\left\|\overline{\mathrm{W}}-\overline{\mathrm{W}}_{\infty}\right\|_{L^{\infty}}^{2}$ from Sobolev norms in molecular coordinates. We indeed have to establish that $\left\|\overline{\mathrm{W}}-\overline{\mathrm{W}}_{\infty}\right\|_{L^{\infty}}^{2}$ remains $\mathcal{O}\left(\epsilon_{\mathrm{M}}^{2}\right)$ if it is the case at initial time. In order to achieve such a control it is necessary to combine the scaling law for derivatives $\int_{\mathbb{R}^{n}} \tilde{\gamma}^{[k]} d \bar{x}=\mathcal{O}\left(\epsilon_{\mathrm{K}}{ }^{2 k+2-n}\right)$ together with scale invariant interpolation inequalities. Upon using the scaling law for the integrals $\int_{\mathbb{R}^{n}} \tilde{\gamma}^{[k]} d \bar{x}$ and assuming that the initial state is $\mathcal{O}\left(\epsilon_{\mathrm{M}}^{2}\right)$ it is then possible to cancel the $\epsilon_{\mathrm{K}}^{-n}$ factor and to establish that $\left\|\overline{\mathrm{W}}-\overline{\mathrm{W}}_{\infty}\right\|_{L^{\infty}}$ effectively remains $\mathcal{O}\left(\epsilon_{\mathrm{M}}^{2}\right)$.

Proposition 5.1. Let $l \geq[n / 2]+2$ be an integer, and let $\overline{\mathrm{V}}$ be a smooth solution of the incompressible NavierSokes equations (20)-(22) over $\left[0, \bar{t}_{1}\right] \times \mathbb{R}^{n}$. Let $\mathrm{A}>0, \xi>0$, and assume that the initial state satisfies the conditions

$$
\int_{\mathbb{R}^{n}} \tilde{\gamma}_{0}^{[k]} d \bar{x} \leq \xi^{2 k+2-n} \mathrm{~A}, \quad 0 \leq k \leq l,
$$

where $\tilde{\gamma}_{0}^{[k]}$ denotes the functional $\tilde{\gamma}^{[k]}$ evaluated at initial time $t=0$. There exist constants $\mathrm{A}_{0}\left(l, n, T_{\min }\right)$ and $\bar{\delta}\left(l, n, T_{\min }\right)$ such that if $\mathrm{A} \leq \mathrm{A}_{0}$ and $\mathrm{A} \xi^{2} \leq \bar{\delta}$, whenever the initial conditions satisfy (60), then for all time $t \in\left[0, t_{1}\right]$ we have

$$
\int_{\mathbb{R}^{n}} \tilde{\gamma}^{[k]} d \bar{x} \leq 2 \xi^{2 k+2-n} \mathrm{~A}, \quad 0 \leq k \leq l .
$$

Moreover we have the estimate

$$
\left\|\overline{\mathrm{w}}-\overline{\mathrm{W}}_{\infty}\right\|_{L^{\infty}}^{2} \leq 2 c_{0} A \xi^{2},
$$

for some contant $c_{0}\left(l, n, T_{\min }\right)$ and we also have $\chi \leq \frac{1}{2} \delta_{\chi}$ where $\delta_{\chi}$ is defined in Corollary 4.\%. 
Proof. We use the scale invariant interpolation inequality valid for $n<2 m$

$$
\|\phi\|_{L^{\infty}} \leq C_{0}\left\|\partial_{\bar{x}}^{m} \phi\right\|_{L^{2}}^{\frac{n}{2 m}}\|\phi\|_{L^{2}}^{1-\frac{n}{2 m}}
$$

where $C_{0}$ depends on $n$ and $m$ and the estimate

$$
\left\|\overline{\mathrm{w}}-\overline{\mathrm{W}}_{\infty}\right\|_{L^{2}}^{2} \leq \widehat{C}_{0} \int_{\mathbb{R}^{n}} \tilde{\gamma}^{[0]} d \bar{x}
$$

where $\widehat{C}_{0}$ only depends of $T_{\text {min }}$. Combining (63) and (64) with $m=l$ we deduce that there exists a constant $c_{0}\left(l, n, T_{\min }\right)$ such that

$$
\left\|\overline{\mathrm{W}}-\overline{\mathrm{W}}_{\infty}\right\|_{L^{\infty}}^{2} \leq c_{0}\left(\int_{\mathbb{R}^{n}} \tilde{\gamma}^{[l]} d \bar{x}\right)^{\frac{n}{2 l}}\left(\int_{\mathbb{R}^{n}} \tilde{\gamma}^{[0]} d \bar{x}\right)^{1-\frac{n}{2 l}} .
$$

From this inequality we obtain that at $t=0$

$$
\left\|\overline{\mathrm{W}}-\overline{\mathrm{W}}_{\infty}\right\|_{L^{\infty}}^{2} \leq c_{0}\left(\xi^{2 l+2-n} \mathrm{~A}\right)^{\frac{n}{2 l}}\left(\xi^{2-n} \mathrm{~A}\right)^{1-\frac{n}{2 t}} \leq c_{0} \mathrm{~A} \xi^{2},
$$

so that initially $\chi^{2}(0) \leq c_{0} \mathrm{~A} \xi^{2}$. On the other hand when $\chi<\delta_{\chi}\left(l, n, T_{\min }\right)$ we also have entropic estimates similar to the estimates (48) and (52) but expressed in molecular coordinates, where $\underline{b}=\min \left(\underline{b}_{0}, \underline{\mathfrak{a}}\right)$ and $c=c\left(l, n, T_{\min }\right)$

$$
\begin{gathered}
\partial_{\bar{t}} \int_{\mathbb{R}^{n}} \tilde{\gamma}^{[0]} d \bar{x}+\underline{b} \int_{\mathbb{R}^{n}} T^{\varkappa} \tilde{\gamma}^{[1]} d \bar{x} \leq 0, \\
\partial_{\bar{t}} \int_{\mathbb{R}^{n}} \tilde{\gamma}^{[k]} d \bar{x}+\underline{b} \int_{\mathbb{R}^{n}} T^{\varkappa} \tilde{\gamma}^{[k+1]} d \bar{x} \leq c \chi^{2} \int_{\mathbb{R}^{n}} T^{\varkappa} \tilde{\gamma}^{[k]} d \bar{x}, \quad 1 \leq k \leq l .
\end{gathered}
$$

We denote by $\chi_{\max }$ a maximum value of $\chi$ over a domain $[0, s] \times \mathbb{R}^{n}$ for some $s>0$. Upon integrating (67) (68) with respect to the time variable over $[0, s]$, and by combining the resulting inequalities, it is easily shown that for $0 \leq k \leq l$ and $0 \leq \bar{t} \leq s$ we have for $\bar{c}=c / \underline{b}$

$$
\int_{\mathbb{R}^{n}} \tilde{\gamma}^{[k]} d \bar{x} \leq \int_{\mathbb{R}^{n}} \tilde{\gamma}_{0}^{[k]} d \bar{x}+\bar{c} \chi_{\max }^{2} \int_{\mathbb{R}^{n}} \tilde{\gamma}_{0}^{[k-1]} d \bar{x}+\cdots+\left(\bar{c} \chi_{\max }^{2}\right)^{k} \int_{\mathbb{R}^{n}} \tilde{\gamma}_{0}^{[0]} d \bar{x} .
$$

Since $\chi^{2}(0) \leq c_{0} \mathrm{~A} \xi^{2}$ we may assume that $\chi_{\max }^{2} \leq 3 c_{0} \mathrm{~A} \xi^{2}$ over some time interval and further assuming that $3 c_{0} \mathrm{~A} \xi^{2} \leq\left(\frac{1}{2} \delta_{\chi}\right)^{2}$ we deduce from the entropic estimates (69) that

$$
\int_{\mathbb{R}^{n}} \tilde{\gamma}^{[k]} d \bar{x} \leq \mathrm{A} \xi^{2 k+2-n}\left(1+3 \bar{c} c_{0} \mathrm{~A}+\cdots+\left(3 \bar{c} c_{0} \mathrm{~A}\right)^{k}\right), \quad 0 \leq k \leq l .
$$

From (65) we obtain that over the time interval $[0, s]$

$$
\chi_{\max }^{2} \leq\left\|\overline{\mathrm{W}}-\overline{\mathrm{W}}_{\infty}\right\|_{L^{\infty}}^{2} \leq c_{0} \mathrm{~A} \xi^{2}\left(1+3 \bar{c} c_{0} \mathrm{~A}+\cdots+\left(3 \bar{c} c_{0} \mathrm{~A}\right)^{l}\right)^{\frac{n}{2 l}} .
$$

Assuming now that $\mathrm{A}_{0}$ is small enough so that for any $\mathrm{A} \leq \mathrm{A}_{0}$ we have

$$
c_{0} \mathrm{~A}\left(1+3 \bar{c} c_{0} \mathrm{~A}+\cdots+\left(3 \bar{c} c_{0} \mathrm{~A}\right)^{l}\right) \leq 2 c_{0} \mathrm{~A},
$$

we obtain that $\chi_{\max }^{2} \leq 2 c_{0} \mathrm{~A} \xi^{2}$ over $[0, s]$. Defining

$$
E=\left\{s \in\left(0, \bar{t}_{1}\right], \forall \hat{s} \in[0, s],\left\|\overline{\mathrm{W}}-\overline{\mathrm{W}}_{\infty}\right\|_{L^{\infty}}^{2} \leq 3 c_{0} \mathrm{~A} \xi^{2}\right\}
$$


and assuming that $3 c_{0} \mathrm{~A} \xi^{2}<\left(\frac{1}{2} \delta_{\chi}\right)^{2}$ it is easily established that $E$ is not empty and $\sup E=\bar{t}_{1}$. Therefore we may set $\bar{\delta}\left(l, n, T_{\min }\right)=\left(1 / 12 c_{0}\right) \delta_{\chi}^{2}$ and we finally have $\left\|\overline{\mathrm{W}}-\overline{\mathrm{W}}_{\infty}\right\|_{L^{\infty}}^{2} \leq 2 c_{0} \mathrm{~A} \xi^{2}$.

We now obtain a global existence theorem by combining the estimates of Proposition 5.1 with a local existence result obtained in $[10,11]$.

Théorème 5.2. Let $n \geq 2$ and $l \geq[n / 2]+2$ be integers and assume that $T_{0}$ and $\bar{v}_{0}$ satisfy $T_{\min } \leq \inf _{\mathbb{R}^{n}} T_{0}$, $\partial_{x} \cdot \bar{v}_{0}=0, \overline{\mathrm{v}}_{0}-\overline{\mathrm{v}}_{\infty} \in H^{k}, k \in \mathbb{N}$. Let $\mathrm{A}>0, \xi>0$, and assume that

$$
\int_{\mathbb{R}^{n}} \tilde{\gamma}_{0}^{[k]} d \bar{x} \leq \xi^{2 k+2-n} \mathrm{~A}, \quad 0 \leq k \leq l
$$

where $\widetilde{\gamma}_{0}^{[k]}$ denotes the functional $\widetilde{\gamma}^{[k]}$ evaluated at initial time $t=0$. There exist constants $\mathrm{A}_{0}\left(l, n, T_{\min }\right)>0$ and $\bar{\delta}\left(l, n, T_{\min }\right)>0$ such that for $\mathrm{A} \leq \mathrm{A}_{0}$ and $\mathrm{A} \xi^{2} \leq \bar{\delta}$ there exists a unique global solution $\overline{\mathrm{V}}=(\bar{w}, T)$ such that

$$
\left\{\begin{array}{l}
\overline{\mathrm{V}}-\overline{\mathrm{V}}_{\infty}, \overline{\mathrm{W}}-\overline{\mathrm{W}}_{\infty} \in C\left([0, \infty), H^{l}\left(\mathbb{R}^{n}\right)\right) \cap C^{1}\left([0, \infty), H^{l-2}\left(\mathbb{R}^{n}\right)\right), \\
\partial_{x} \overline{\mathrm{V}}, \partial_{x} \overline{\mathrm{W}} \in L^{2}\left((0, \infty), H^{l}\left(\mathbb{R}^{n}\right)\right),
\end{array}\right.
$$

and we have the estimates

$$
\int_{\mathbb{R}^{n}} \tilde{\gamma}^{[k]} d \bar{x} \leq 2 \xi^{2 k+2-n} \mathrm{~A}, \quad 0 \leq k \leq l,
$$

Furthermore, this solution is smooth and we have

$$
\lim _{\bar{t} \rightarrow \infty}\left\|\overline{\mathrm{V}}(\bar{t}, \cdot)-\overline{\mathrm{V}}_{\infty}\right\|_{L^{\infty}}=0
$$

Proof. We select the constants $\mathrm{A}_{0}\left(l, n, T_{\min }\right)>0$ and $\bar{\delta}\left(l, n, T_{\min }\right)>0$ of Proposition 5.1. These value will indeed insure that $\chi$ remains small with $\chi \leq \frac{1}{2} \delta_{\chi}$. Corresponding to this value of $\bar{\delta}$, we have estimates in the forms $\left\|\mathrm{W}-\mathrm{W}_{\infty}\right\|_{L^{\infty}}^{2} \leq 2 c_{0} \mathrm{~A} \xi^{2}$. We now select $\mathrm{M}_{\bar{w}}>0$ and $\mathrm{M}_{\tau}>0$ large enough such that

$$
\left\{z \in \mathbb{R}^{n+1} ;\left\|z-\overline{\mathrm{W}}_{\infty}\right\| \leq\left(2 c_{0} \mathrm{~A}\right)^{1 / 2} \xi+1\right\} \subset\left(-\mathrm{M}_{\bar{w}}, \mathrm{M}_{\bar{w}}\right)^{n} \times\left(-\mathrm{M}_{\tau}, \mathrm{M}_{\tau}\right)
$$

we define $\widetilde{\mathcal{O}}_{0}=\left(-\mathrm{M}_{\bar{w}}, \mathrm{M}_{\bar{w}}\right)^{n} \times\left(-\mathrm{M}_{\tau}, \mathrm{M}_{\tau}\right), \mathcal{O}_{0}=\mathcal{F}^{-1}\left(\widetilde{\mathcal{O}}_{0}\right)$, where $\mathcal{F}: \mathbb{R}^{n} \times(0, \infty) \rightarrow \mathbb{R}^{n+1}$ is given by $\mathcal{F}(\mathrm{v}, \mathrm{T})=$ $(\mathrm{v} / \sqrt{\mathrm{T}}, \log \mathrm{T})$, and one can show that $\mathcal{O}_{0}$ is convex [11]. Let then $0<d_{1}<d\left(\overline{\mathcal{O}}_{0}, \partial \mathcal{O}_{\overline{\mathrm{v}}}\right)$, and define the open sets $\mathcal{O}_{1}=\left\{z \in \mathcal{O}_{\overline{\mathrm{V}}} ; d\left(z, \mathcal{O}_{0}\right)<d_{1}\right\}$ and $\widetilde{\mathcal{O}}_{1}=\mathcal{F}\left(\mathcal{O}_{1}\right)$. For functions taking their values in $\mathcal{O}_{1}$ we have inequalities in the form $\left\|\bar{\nabla}-\bar{\nabla}_{\infty}\right\|_{H^{l}} \leq m\left\|\overline{\mathrm{W}}-\overline{\mathrm{W}}_{\infty}\right\|_{H^{l}}$ where $m$ only depends on $l$ and $\mathcal{O}_{1}[11,16]$. We thus obtain a priori estimates in the form $\left\|\overline{\mathrm{V}}-\overline{\mathrm{V}}_{\infty}\right\|_{H^{l}} \leq C(\mathrm{~A}, \xi)$ where $C(\mathrm{~A}, \xi)^{2}=2 m^{2}\left(1+\widehat{C}_{0}\right) \sum_{0 \leq i \leq l} \xi^{2 i+2-n} \mathrm{~A}$. We then set $b=C(\mathrm{~A}, \xi)+1$ and from the local existence theorem established in [11] we have local solutions over a time interval $\left[0, \bar{t}_{1}\right]$ built with the paramaters $\mathcal{O}_{0}, d_{1}$, and $b$. It is important to note that the parameter $b$ may be large because the norms $\int_{\mathbb{R}_{n}^{n}} \tilde{\gamma}^{[k]} d \bar{x}$ in molecular coordinates scale like $2 \xi^{2 k+2-n} \mathrm{~A}$, so that $\bar{t}_{1}$, which depends on A and $\xi$, will be smaller as $\xi$ gets smaller. However, in order to establish a global existence theorem, we can use a different local existence time $\bar{t}_{1}$ for each fixed values of A and $\xi$.

Let $\overline{\mathrm{V}}=\left(\bar{v}_{0}, T_{0}\right)$ satisfy $T_{\min } \leq \inf _{\mathbb{R}^{n}} T_{0}, \partial_{x} \cdot \bar{v}_{0}=0, \overline{\mathrm{v}}_{0}-\overline{\mathrm{v}}_{\infty} \in H^{k}, k \in \mathbb{N}$, and $\int_{\mathbb{R}^{n}} \tilde{\gamma}_{0}^{[k]} d \bar{x} \leq \xi^{2 k+2-n} \mathrm{~A}$ for $0 \leq k \leq l$. Then by construction $\overline{\mathrm{V}}_{0} \in \mathcal{O}_{0}$ and $\left\|\mathrm{v}-\mathrm{v}_{\infty}\right\|_{H^{l}}<b$, and we have a local solution over the time interval $\left[0, \bar{t}_{1}\right]$. It is then straightforward to establish that $\|\overline{\mathrm{W}}-\overline{\mathrm{W}}\|_{L^{\infty}}^{2}$ remains smaller that $2 c_{0} \mathrm{~A} \xi^{2}$ over $\left[0, \bar{t}_{1}\right]$ and that the estimates of Proposition 5.1 hold. In particular we have $\overline{\mathrm{v}}\left(\bar{t}_{1}\right) \in \mathcal{O}_{0}$ and $\left\|\overline{\mathrm{v}}\left(\bar{t}_{1}\right)-\overline{\mathrm{v}}_{\infty}\right\|_{H^{l}}<b$. We may now use again the local existence theorem over $\left[\bar{t}_{1}, 2 \bar{t}_{1}\right]$ and the estimates of Proposition 5.1 hold on $\left[0,2 \bar{t}_{1}\right]$. An easy induction shows that the solution is a global solution. 
In order to establish asymptotic stability we let $\Phi(\bar{t})=\int_{\mathbb{R}^{n}} \tilde{\gamma}^{[l-1]} d \bar{x}=\int_{\mathbb{R}^{n}}\left\|\partial_{\bar{x}}^{l-1} \overline{\mathrm{W}}\right\|^{2} d \bar{x}$ and from the equations governing higher order entropies it is easily checked that there exists a constant $C\left(l, n, T_{\min }\right)$ such that

$$
\xi^{2} \int_{0}^{\infty}|\Phi(\bar{t})| d \bar{t}+\int_{0}^{\infty}\left|\partial_{\bar{t}} \Phi(\bar{t})\right| d \bar{t} \leq C\left(l, n, T_{\min }\right) \xi^{2 l-n} \mathrm{~A} .
$$

As a consequence, we have $\Phi(\bar{t}) \rightarrow 0$ as $\xi^{2} \bar{t} \rightarrow \infty$ and asymptotic stability is then a consequence of the interpolation inequality $\|\phi\|_{L^{\infty}} \leq C_{0}\left\|\partial_{x}^{l-1} \phi\right\|_{L^{2}}^{a}\|\phi\|_{L^{2}}^{1-a}$ where $a=n / 2(l-1)$ and the proof is complete.

\subsection{Global existence for small Mach and Knudsen numbers}

In this section, we investigate global existence of solutions for small Mach and Kundsen numbers.

Théorème 5.3. Let $n \geq 2$ and $l \geq[n / 2]+2$ be integers. Assume that the coefficients $\lambda$ and $\eta$ satisfy (23) with $\varkappa \geq 1 / 2$ and that $a_{k}=1$, for $k \geq 0$. Assume that $T_{0}$ and $\bar{v}_{0}$ satisfying $T_{\min } \leq \inf _{\mathbb{R}^{n}} T_{0}, \partial_{x} \cdot \bar{v}_{0}=0$, $\overline{\mathrm{V}}_{0}-\mathrm{v}_{\infty} \in H^{k}, k \in \mathbb{N}$. Let $\epsilon_{\mathrm{K}}>0, \operatorname{Re}>0, \mathrm{~A}>0$ and assume that

$$
\int_{\mathbb{R}^{n}} \tilde{\gamma}_{0}^{[k]} d x \leq \epsilon_{\mathrm{K}}^{2 k+2} \operatorname{Re}^{2} \mathrm{~A}, \quad 0 \leq k \leq l,
$$

where $\widetilde{\gamma}_{0}^{[k]}$ denotes the functional $\widetilde{\gamma}^{[k]}$ evaluated at initial time $t=0$. There exist constants $\mathrm{A}_{0}\left(l, n, T_{\min }\right)>0$ and $\bar{\delta}\left(l, n, T_{\min }\right)>0$ such that for $\operatorname{Re}^{2} \mathrm{~A} \leq \mathrm{A}_{0}$ and $\operatorname{Re}^{2} \epsilon_{\mathrm{K}}^{2} \mathrm{~A} \leq \bar{\delta}$ there exists a unique global solution $\overline{\mathrm{V}}=(\bar{v}, T)$ such that

$$
\left\{\begin{array}{l}
\overline{\mathrm{V}}-\overline{\mathrm{V}}_{\infty}, \overline{\mathrm{W}}-\overline{\mathrm{W}}_{\infty} \in C\left([0, \infty), H^{l}\left(\mathbb{R}^{n}\right)\right) \cap C^{1}\left([0, \infty), H^{l-2}\left(\mathbb{R}^{n}\right)\right), \\
\partial_{x} \overline{\mathrm{V}}, \partial_{x} \overline{\mathrm{W}} \in L^{2}\left((0, \infty), H^{l}\left(\mathbb{R}^{n}\right)\right),
\end{array}\right.
$$

and we have the estimates

$$
\int_{\mathbb{R}^{n}} \tilde{\gamma}^{[k]} d x \leq 2 \epsilon_{\mathrm{K}}^{2 k+2} \operatorname{Re}^{2} \mathrm{~A}, \quad 0 \leq k \leq l,
$$

and for some constant $c_{0}\left(l, n, T_{\min }\right)$

$$
\left\|\overline{\mathrm{W}}-\overline{\mathrm{W}}_{\infty}\right\|_{L^{\infty}}^{2} \leq 2 c_{0} \epsilon_{\mathrm{K}}^{2} \operatorname{Re}^{2} \mathrm{~A} .
$$

Furthermore, this solution is smooth and we have $\lim _{t \rightarrow \infty}\left\|\overline{\mathrm{V}}(t, \cdot)-\overline{\mathrm{V}}_{\infty}\right\|_{L^{\infty}}=0$.

Proof. Theorem 5.3 is a consequence of Theorem 5.2 with $\xi=\epsilon_{\mathrm{K}}$ and A replaced by $\operatorname{Re}^{2} \mathrm{~A}$. In addition, if we set $\Psi(t)=\int_{\mathbb{R}^{n}}\left\|\partial_{x}^{l-1} \overline{\mathrm{W}}\right\|^{2} d x$ we can rewrite 78 in the form

$$
\int_{0}^{\infty}|\Psi(t)| d t+\int_{0}^{\infty}\left|\partial_{t} \Psi(t)\right| d t \leq C\left(l, n, T_{\min }\right) \epsilon_{\mathrm{K}}^{2} \mathrm{~A},
$$

and the convergence to $\bar{\nabla}_{\infty}$ holds on a macroscopic time scale.

Remarque 5.4. Letting $\tau-\tau_{\infty}=\epsilon_{\mathrm{M}} \theta$ and $\bar{v}=\epsilon_{\mathrm{M}} v$ one may also use the variables $(v, \theta)$ with the macroscopic coordinates $(t, x)$ but using the variables $(\bar{w}, \tau)$ in molecular coordinates $(\bar{t}, \bar{x})$ seems more natural. In addition, we have only used that $\operatorname{Re}^{2} \mathrm{~A}$ is small in order to obtain a stable domain so that there is room for improvement.

Remarque 5.5. The methods developped for incompressible flows can be adapted mutatis mutandis to the situation of compressible or zero Mach number flows. 


\section{REFERENCES}

[1] L. Boltzmann, Vorlesungen über Gastheorie, Vol. I, Leipzig, (1895). Translated by G. Brush, Lectures on gas Theory, Berkeley, University of California Press 1964.

[2] C. Cercignani, The Boltzmann Equation and Its Applications, Applied Mathematical Sciences, Volume 67, Springer-Verlag 1988.

[3] S. Chapman and T. G. Cowling, The Mathematical Theory of Non-Uniform Gases, Applied Mathematical Sciences, Cambridge University Press, Cambridge 1970.

[4] L. Comtet, Analyse Combinatoire, Tomes I et II, Presses Universitaires de France, Paris 1970.

[5] L. Desvillettes and C. Villani, On the Trend to Global Equilibrium for Spatially Inhomogeneous Kinetic Systems: the Boltzmann Equation, Inventiones Mathematicae 159 (2005) 245-316.

[6] L. C. Evans, A Survey of Entropy Methods for Partial Differential Equations, Bulletin of the AMS 41 (2004) $409-438$.

[7] J. H. Ferziger, H. G. Kaper, Mathematical theory of transport processes in gases, North-Holland, 1972.

[8] J. Garcia-Cuerva and J.L. Rubio de Francia, Weighted Norm Inequalities and Related Topics, North Holland Mathematical Studies 116, Elsevier Science Publisher, Amsterdam 1985.

[9] V. Giovangigli, Multicomponent flow modeling, Birkhäuser, Boston 1999. Erratum at http: \\cmap.polytechnique.fr ggiovangi $^{2}$

[10] V. Giovangigli, Entropies d'Ordre Supérieur. C. R. Acad. Sci. Paris, Ser. I, 343 (2006) 179-184.

[11] V. Giovangigli, Higher Order Entropies, Archive for Rational Mechanical Analysis, (accepted for publication) (2006). See also Rapport Interne CMAP 592.

[12] V. Giovangigli, Higher Order Entropies for Compressible Fluid Models, In preparation, (2007).

[13] V. Giovangigli and M. Massot, Asymptotic Stability of Equilibrium States for Multicomponent Reactive Flows, Mathematical Models \& Methods in Applied Science, 8 (1998) 251-297.

[14] F. Golse, From Kinetic to Macroscopic Models, in : Kinetic Equations and Asymptotic Theory, B. Perthame and L. Desvillettes eds, Series in Applied Mathematics, Gauthier-Villars/Elseviers, (2000).

[15] F. John and L. Nirenberg, On Functions of Bounded Mean Oscillation, Comm. Pure Appl. Math., XIV (1961) $415-426$.

[16] S. Kawashima, Systems of a Hyperbolic-Parabolic Composite Type, with applications to the equations of Magnetohydrodynamics, Doctoral Thesis, Kyoto University 1984.

[17] O.A. Ladyz̃enskaja, V.A. Solonikov, and N.N. Ural'ceva, Linear and Quasilinear Equations of Parabolic Type, Translations of Mathematical Monographs, 23, American Mathematical Society, Providence, Rhode Island, (1968).

[18] P. L. Lions, Mathematical Topics in Fluid Mechanics, Volumes 1 and 2, Oxford Lecture Series in Mathematics and its Applications, Oxford 1996 and 1998.

[19] Y. Meyer, Ondelettes et Opérateurs, Volume 1: Ondelettes, and Volume 2: Opérateurs de Calderón-Zygmund, Hermann, Paris 1990.

[20] Y. Meyer et R. Coifman, Ondelettes et Opérateurs, Volume 3: Opérateurs Multilinéaires, Hermann, Paris 1991.

[21] T.Y. Ting, Generalized Korn's Inequalities, Tensor, 25 (1972) 295-302.

[22] C. Villani, Entropy Dissipation and Convergence to Equilibrium, Course at Institut Henri Poincaré, (2001). 